\title{
The Adaptive Dynamics of Community Structure
}

\author{
Ulf Dieckmann, Åke Brännström, \\ Reinier HilleRisLambes, and Hiroshi C. Ito
}

\subsection{Introduction}

Attempts at comprehending the structures of ecological communities have a long history in biology, reaching right back to the dawn of modern ecology. A seminal debate allegedly occurred between early-twentieth-century plant ecologists Frederic E. Clements and Henry A. Gleason. Textbooks have it (e.g., Calow 1998: 145) that Clements viewed ecological communities as being structured by rich internal dependencies, akin to organisms (Clements 1916), while Gleason held that members of ecological communities were relatively independent of each other, filling ecological niches provided by the abiotic environment (Gleason 1926). While the actual approaches of these two luminaries of plant ecology were more complex than this well-worn caricature suggests (Eliot, in press), their purported positions conveniently established an important conceptual continuum for the mechanistic interpretation of community structures observed in nature.

Modern echoes of this old debate can be found in notions of niche construction (Odling-Smee et al. 2003), leaning towards the Clementsian end of the spectrum, or in the neutral theory of biodiversity and biogeography (Hubbell 2001), which is more in line with a Gleasonian perspective. Like in many other fundamental disputes in ecology, neither side turns out to be simply right or wrong. Instead, disagreements of this kind tend to be resolved at a higher level - by recognizing, firstly, that the original controversy was based on unduly generalized and polarized claims, and secondly, by refocusing scientific attention on elucidating the specific factors and mechanisms that push ecological systems towards either end of the intermediary continuum. Below we will propose such an overarching notion for reinterpreting the Clements-Gleason debate.

Early theoretical models of community structure were based on the simplifying concept of randomly established ecological communities (May 1973). This first wave of models suggested that larger random communities were less likely to possess stable fixed-point equilibria than smaller ones - thus giving 
rise to yet another long-lasting debate in ecology, about the relationship between community complexity or diversity on the one hand, and community stability or productivity on the other (e.g., Elton 1958; McCann 2000). A second wave of models subsequently imbued such investigations with a higher degree of ecological realism by accounting for the historical route through which new ecological communities are assembled from scratch, and considering more than only infinitely small community perturbations (Post and Pimm 1983; Drake 1990; Law 1999). These assembly models usually relied on the notion of a species pool from which individual species are drawn successively and at random, mimicking the arrival of immigrants from outside an incipient community. A third, much more recent, wave of models rises above considering mere immigration from such a pre-defined species pool, by trying to understand the potential of natural selection for shaping the dynamics and structures of ecological communities (Caldarelli et al. 1998; Drossel et al. 2001; Loeuille and Loreau 2005; Ito and Ikegami 2003, 2006). Together, these alternative suites of models suggest that community structures in ecology can only be fully comprehended when processes of interaction (first-wave models), immigration (second-wave models), and adaptation (third-wave models) are taken into account. Appreciating the mechanisms that generate and maintain diversity in ecological communities thus requires methods stretching across the typically different time scales of interactions, immigrations, and adaptations.

Once the dynamics of community formation are recognized to encompass phenotypic adaptation, it is instructive to recast the classic ClementsGleason debate in terms of fitness landscapes. Under frequency- and densityindependent selection, the fitness landscapes experienced by members of an ecological community are independent of the community's composition, directly corresponding to a Gleasonian view. The resultant constant fitness landscapes result in what is known as 'optimizing selection'. By contrast, when the fitness of community members depend on their overall density and individual frequency, fitness landscapes vary with a community's composition. A situation in which this variability is very pronounced, and the frequency- and density-independent components of selection pressures within the community accordingly are relatively weak, neatly corresponds to a Clementsian view. As so often, reality is bound to lie in between these two extremes.

Consequently, an evolutionary perspective on community ecology sheds new light on two fundamental ecological debates. On the one hand, assessing the degree to which fitness landscapes are varying with community composition provides a practical approach for locating specific communities along the Clements-Gleason continuum. On the other hand, evolutionary dynamics literally add new dimensions to concepts of community stability: community structures that are ecologically stable are unlikely also to be evolutionarily stable. This realization challenges earlier conclusions as to how the stability of communities is affected by their complexity or diversity. In particular, 
ecologically unstable communities may be stabilized by the fine-tuning afforded through coevolutionary adaptations, while ecologically stable communities may be destabilized by evolutionary processes such as arms races, taxon cycles, speciation, and selection-driven extinctions.

In the time-honored quest for understanding community structures, ecology and evolution are thus linked inevitably and intricately, with frequencyand density-dependent selection pressures playing important roles. This sets the stage for considering the utility of adaptive dynamics theory for understanding community structure. Adaptive dynamics theory is a conceptual framework for analyzing the density- and frequency-dependent evolution of quantitative traits, based on a general approach to deriving fitness functions, selection pressures, and evolutionary dynamics from the underlying ecological interactions and population dynamics (e.g., Metz et al. 1992; Dieckmann 1994; Metz et al. 1996; Dieckmann and Law 1996; Geritz et al. 1997, 1998). After introducing the main concepts and models of this approach in Sect. 8.2, this chapter proceeds, in Sect. 8.3 and 8.4, to brief discussions of how selection pressures may drive the increase or decrease, respectively, of species numbers in ecological communities. Armed with this general background, four specific examples of community evolution models are studied in Sects. 8.5 to 8.8 .

Models of evolutionary community assembly are still in their infancy. Accordingly, much room currently exists for investigating systematic variations of already proposed model structures, so as to separate critical from incidental model assumptions and ingredients. The main purpose of this chapter is to introduce readers to a particularly versatile mathematical toolbox for carrying out these much-needed future investigations.

\subsection{Models of adaptive dynamics}

The theory of adaptive dynamics derives from considering ecological interactions and phenotypic variation at the level of individuals. Extending classical birth and death processes through mutation, adaptive dynamics models keep track, across time, of the phenotypic composition of populations in which trait values of offspring are allowed to differ from those of their parents.

Throughout this chapter we will adhere to the following notation. Time is denoted by $t$. The number of species in the considered community is $N$. The values of quantitative traits in species $i$ are denoted by $x_{i}$, be they univariate or multivariate. The abundance of individuals with trait value $x_{i}$ is denoted by $n_{i}\left(x_{i}\right)$, while $n_{i}$ denotes the total abundance of individuals in species $i$. If species $i$ harbors individuals with $m_{i}$ distinct trait values $x_{i k}$, its phenotypic density is given by $p_{i}\left(x_{i}\right)=\sum_{k=1}^{m_{i}} n_{i}\left(x_{i k}\right) \delta\left(x_{i}-x_{i k}\right)=\sum_{k=1}^{n_{i}} \delta\left(x_{i}-x_{i k}\right)$, where $\delta$ denotes Dirac's delta function. A species with $m_{i}=1$ is said to be monomorphic. For small $m_{i}$, species $i$ may be characterized as being oligomorphic; when $m_{i}$ is large, it will be called polymorphic. The community's 
phenotypic composition is described by $p=\left(p_{1}, \ldots, p_{N}\right)$. The per capita birth and death rates of individuals with trait value $x_{i}^{\prime}$ in a community with phenotypic composition $p$ are denoted by $b_{i}\left(x_{i}^{\prime}, p\right)$ and $d_{i}\left(x_{i}^{\prime}, p\right)$. Reproduction is clonal, mutant individuals arise with probabilities $\mu_{i}\left(x_{i}\right)$ per birth event, and their trait values $x_{i}^{\prime}$ are drawn from distributions $M_{i}\left(x_{i}^{\prime}, x_{i}\right)$ around parental trait values $x_{i}$.

If all species in the community are monomorphic, with resident trait values $x=\left(x_{1}, \ldots, x_{N}\right)$, and if their ecological dynamics attain an equilibrium attractor, with resident abundances $\bar{n}_{i}(x)$, the resultant phenotypic composition is denoted by $\bar{p}(x)$. The per capita birth, death, and growth rates of individuals with trait value $x_{\underline{i}}^{\prime}$ will then be given by $\bar{b}_{i}\left(x_{i}^{\prime}, x\right)=b_{i}\left(x_{i}^{\prime}, \bar{p}(x)\right)$, $\bar{d}_{i}\left(x_{i}^{\prime}, x\right)=d_{i}\left(x_{i}^{\prime}, \bar{p}(x)\right)$, and $\bar{f}_{i}\left(x_{i}^{\prime}, x\right)=\bar{b}_{i}\left(x_{i}^{\prime}, x\right)-\bar{d}_{i}\left(x_{i}^{\prime}, x\right)$, respectively. In adaptive dynamics theory, the latter quantity is called invasion fitness. For a mutant $x_{i}^{\prime}$ to have a chance of invading a resident community $x$, its invasion fitness needs to be positive. The notion of invasion fitness $\bar{f}_{i}\left(x_{i}^{\prime}, x\right)$ makes explicit that the fitness $\bar{f}_{i}$ of individuals with trait values $x_{i}^{\prime}$ can only be evaluated relative to the environment in which they live, which, in the presence of density- and frequency-dependent selection, depends on $x$. Invasion fitness can be calculated also for more complicated ecological scenarios, for example, when species exhibit physiological population structure, when they experience non-equilibrium ecological dynamics, or when they are exposed to fluctuating environments (Metz et al. 1992). If a community's ecological dynamics possess several coexisting attractors, invasion fitness will be multi-valued. While strictly monomorphic populations will seldom be found in nature, it turns out that the dynamics of polymorphic populations can often be well approximated and understood in terms of the simpler monomorphic cases. For univariate traits, depicting the sign structure of invasion fitness results in so-called pairwise invasibility plots (Matsuda 1985; van Tienderen and de Jong 1986, Metz et al. 1992, 1996; Kisdi and Meszéna 1993; Geritz et al. 1997).

Derivatives of invasion fitness help to understand the course and outcome of evolution. The selection pressure $g_{i}(x)=\left.\frac{\partial}{\partial x_{i}^{\prime}} \bar{f}_{i}\left(x_{i}^{\prime}, x\right)\right|_{x_{i}=x_{i}^{\prime}}$ acting on trait value $x_{i}$ is given by the local slope of the fitness landscape $\bar{f}_{i}\left(x_{i}^{\prime}, x\right)$ at $x_{i}^{\prime}=x_{i}$. When $x_{i}$ is multivariate, this derivative is a gradient vector. Selection pressures in multi-species communities are characterized by $g(x)=\left(g_{1}\left(x_{1}\right), \ldots, g_{N}\left(x_{N}\right)\right)$. Trait values $x^{*}$ at which this selection gradient vanishes, $g\left(x^{*}\right)=0$, are called evolutionarily singular (Metz et al. 1992). Also the signs of the second derivatives of invasion fitness at evolutionarily singular trait values reveal important information. When the mutant Hessian $h_{m m, i}\left(x^{*}\right)=\left.\frac{\partial^{2}}{\partial x_{i}^{\prime 2}} \bar{f}_{i}\left(x_{i}^{\prime}, x\right)\right|_{x_{i}^{\prime}=x_{i}^{*}, x=x^{*}}$ is negative definite, $x_{i}^{*}$ is at a fitness maximum, implying (local) evolutionary stability. When $h_{m m, i}\left(x^{*}\right)-h_{r r, i}\left(x^{*}\right)$ is negative definite, where $h_{r r, i}\left(x^{*}\right)=\left.\frac{\partial^{2}}{\partial x_{i}^{2}} \bar{f}_{i}\left(x_{i}^{\prime}, x\right)\right|_{x_{i}^{\prime}=x_{i}^{*}, x=x^{*}}$ denotes the resident Hessian, subsequent invasion steps in the vicinity of $x_{i}^{*}$ will approach $x_{i}^{*}$, implying (strong) convergence stability. 
Based on these considerations, four classes of models are used to investigate the adaptive dynamics of ecological communities at different levels of resolution and generality. Details concerning the derivations of these models are provided in the Appendix and their formal relations are summarized in Fig. 8.2. We now introduce these four model classes in turn.

Individual-based birth-death-mutation processes: polymorphic and stochastic. Under the individual-based model specified above, polymorphic distributions of trait values stochastically drift and diffuse through selection and mutation (Dieckmann 1994; Dieckmann et al. 1995). See Fig. 8.1a for an illustration. Using the specification of the birth, death, and mutation processes provided by the functions $b_{i}, d_{i}, \mu_{i}$, and $M_{i}$, efficient algorithms for this class of models (Dieckmann 1994) will typically employ Gillespie's minimal process method (Gillespie 1976).
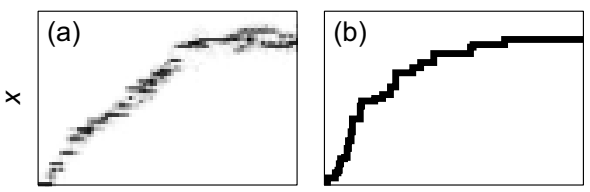

Time, $t$
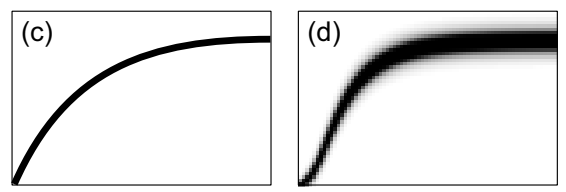

Fig. 8.1. Models of adaptive dynamics. Panel (a) illustrates the individual-based birth-death-mutation process (polymorphic and stochastic), panel (b) shows an evolutionary random walk (monomorphic and stochastic), panel (c) represents the gradient-ascent model (monomorphic and deterministic, described by the canonical equation of adaptive dynamics), and panel (d) depicts an evolutionary reactiondiffusion model (polymorphic and deterministic).

Evolutionary random walks: monomorphic and stochastic. In large populations characterized by low mutation rates, evolution in the individual-based birth-death-mutation process proceeds through sequences of trait substitutions (Metz et al. 1992). During each trait substitution, a mutant with positive invasion fitness quickly invades a resident population, typically ousting the former resident (Geritz et al. 2002). The concatenation of trait substitutions produces the sort of directed random walk depicted in Fig. 8.1b, formally described by the master equation

$$
\frac{\mathrm{d}}{\mathrm{d} t} P(x)=\int\left[r\left(x, x^{\prime}\right) P\left(x^{\prime}\right)-r\left(x^{\prime}, x\right) P(x)\right] \mathrm{d} x^{\prime}
$$

for the probability density $P(x)$ of observing trait value $x$, with probabilistic transition rates

$$
r\left(x^{\prime}, x\right)=\sum_{i=1}^{N} \mu_{i}\left(x_{i}\right) \bar{b}_{i}\left(x_{i}, x\right) M_{i}\left(x_{i}^{\prime}, x_{i}\right) \bar{n}_{i}(x) s_{i}\left(x_{i}^{\prime}, x\right) \prod_{j=1, j \neq i}^{N} \delta\left(x_{j}^{\prime}-x_{j}\right)
$$




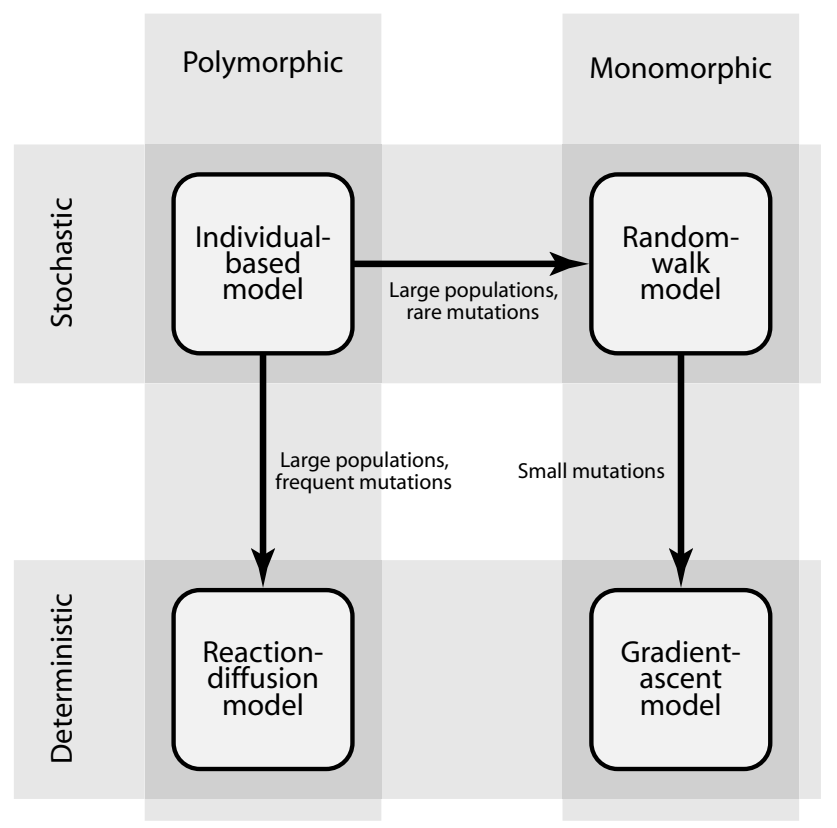

Fig. 8.2. Formal relations between the models of adaptive dynamics. The four classes of model are shown as rounded boxes, and the three derivations as arrows. Arrow labels highlight key assumptions.

(Dieckmann 1994; Dieckmann et al. 1995; Dieckmann and Law 1996). Here $\delta$ again denotes Dirac's delta function, and $s_{i}\left(x_{i}^{\prime}, x\right)=\max \left(0, \bar{f}_{i}\left(x_{i}^{\prime}, x\right)\right) / \bar{b}_{i}\left(x_{i}^{\prime}, x\right)$ is the probability with which the mutant $x_{i}^{\prime}$ survives accidental extinction through demographic stochasticity while still being rare in the large population of resident individuals (e.g., Athreya and Ney 1972). If also the resident population is small enough to be subject to accidental extinction, $s_{i}\left(x_{i}^{\prime}, x\right)=\left(1-e^{-2 \tilde{f}_{i}\left(x_{i}^{\prime}, x\right)}\right) /\left(1-e^{-2 \tilde{f}_{i}\left(x_{i}^{\prime}, x\right) \bar{n}_{i}(x)}\right)$ with $\tilde{f}_{i}\left(x_{i}^{\prime}, x\right)=$ $\left.\bar{f}_{i}\left(x_{i}^{\prime}, x\right)\right) /\left[\bar{b}_{i}\left(x_{i}^{\prime}, x\right)+\bar{d}_{i}\left(x_{i}^{\prime}, x\right)\right]$ provides a more accurate approximation (e.g., Crow and Kimura 1970). The resulting evolutionary random walk models are again typically implemented using Gillespie's minimal process method (Dieckmann 1994).

Gradient-ascent models: monomorphic and deterministic. If mutation steps are not only rare but also small, the dynamics of evolutionary random walks are well approximated by smooth trajectories, as shown in Fig. 8.1c. These trajectories represent the evolutionary random walk's expected path and can be approximated by the canonical equation of adaptive dynamics (Dieckmann 1994; Dieckmann et al. 1995; Dieckmann and Law 1996), which, in its simplest form, is given by

$$
\frac{\mathrm{d}}{\mathrm{d} t} x_{i}=\frac{1}{2} \mu_{i}\left(x_{i}\right) \bar{n}_{i}(x) \sigma_{i}^{2}\left(x_{i}\right) g_{i}(x)
$$

for $i=1, \ldots, N$, where

$$
\sigma_{i}^{2}\left(x_{i}\right)=\int\left(x_{i}^{\prime}-x_{i}\right)^{T}\left(x_{i}^{\prime}-x_{i}\right) M_{i}\left(x_{i}^{\prime}, x_{i}\right) \mathrm{d} x_{i}^{\prime}
$$


is the variance-covariance matrix of the symmetric mutation distribution $M_{i}$ around trait value $x_{i}$. Implementations of this third class of models typically rely on simple Euler integration or on the fourth-order Runge-Kutta method (e.g., Press et al. 1992).

Reaction-diffusion models: polymorphic and stochastic. In large populations characterized by high mutation rates, stochastic elements in the dynamics of the phenotypic distributions become negligible. This enables formal descriptions of reaction-diffusion type (e.g., Kimura 1965; Bürger 1998). Specifically, the reaction-diffusion approximation of the birth-death-mutation process described above is given by

$$
\frac{\mathrm{d}}{\mathrm{d} t} p_{i}\left(x_{i}\right)=f_{i}\left(x_{i}, p\right) p_{i}\left(x_{i}\right)+\frac{1}{2} \sigma_{i}^{2}\left(x_{i}\right) * \frac{\partial^{2}}{\partial x_{i}^{2}} \mu_{i}\left(x_{i}\right) b_{i}\left(x_{i}, p\right) p_{i}\left(x_{i}\right)
$$

for $i=1, \ldots, N$, where $\sigma_{i}^{2}\left(x_{i}\right)$ is the variance-covariance matrix of the symmetric and homogeneous mutation distribution $M_{i}$, and where $*$ denotes the elementwise multiplication of two matrices followed by summation over all resultant matrix elements.. An illustration of reaction-diffusion dynamics is shown in Fig. 8.1d. Models of this fourth class are best implemented using so-called implicit integration methods (e.g., Crank 1975). It ought to be highlighted, however, that the infinitely extended tails that the distributions $p_{i}$ instantaneously acquire in this framework can give rise to artifactual dynamics that offer no good match to the actual dynamics of the underling birth-deathmutation processes in finite populations. The derivation of finite-size corrections to the traditional reaction-diffusion limit overcomes these shortcomings (Dieckmann, unpublished).

At the expense of ignoring genetic intricacies, models of adaptive dynamics are geared to analyzing the evolutionary implications of complex ecological settings. In particular, such models can be used to study all types of densityand frequency-dependent selection, and are equally well geared to describing single-species evolution and multi-species coevolution. As explained above, the four model classes specified in this section are part of a single conceptual and mathematical framework, which implies that switching back and forth between alternative descriptions of any evolutionary dynamics driven by births, deaths, and mutations - as mandated by particular problems in evolutionary ecology - will be entirely straightforward.

\subsection{Selection-driven increases in species numbers}

Frequency-dependent selection is crucial for understanding how selection pressures can increase the number of species within an ecological community:

- First, whenever selection is optimizing, a single type within each species will be most favored by selection, leaving no room for the stable coexistence 
of multiple types per species. Frequency-dependent selection pressures, by contrast, can readily create an 'advantage of rarity,' so that multiple types within a species may be stably maintained: as soon as a type's abundance becomes low, the advantage of rarity boosts its growth rate and thus stabilizes the coexistence.

- Second, whereas gradual evolution under optimizing selection can easily bring about stabilizing selection, it can never lead to disruptive selection. This is because, under optimizing selection, the two relevant notions of stability - evolutionary stability on the one hand (Maynard Smith and Price 1973) and convergence stability on the other (Christiansen 1991) are strictly equivalent: a strategy will be convergence stable if and only if it is evolutionarily stable, and vice versa (e.g., Eshel 1983; Meszéna et al. 2001). Frequency-dependent selection pressures, by contrast, allow for evolutionary branching points, at which directional selection turns disruptive. A gradually evolving population is then trapped at the underlying convergence stable fitness minimum until it splits up into two branches, which subsequently will diverge. This makes the speciation process itself adaptive, and underscores the importance of ecology for understanding speciation.

It is thus clear that frequency-dependent selection is necessary both for the endogenous origin and for the stable maintenance of coexisting types within species.

For univariate traits, the normal form for the invasion fitness of mutants with trait values $x^{\prime}$ in resident populations with trait values $x$ that are close to an evolutionary branching point with trait value $x^{*}=0$ is given by

$$
f\left(x^{\prime}, x\right)=x^{\prime 2}+c x^{2}-(1+c) x^{\prime} x
$$

with $c>1$ (e.g., Dieckmann 1994: 91). From this we can see that the selection pressure at $x^{*}$ ceases, $g\left(x^{*}\right)=0$, that $x^{*}$ is not locally evolutionarily stable, $h_{m m}\left(x^{*}\right)=1>0$, and that $x^{*}$ is convergence stable, $h_{m m}\left(x^{*}\right)-h_{r r}\left(x^{*}\right)=$ $1-c<0$. Under these conditions, trait substitutions in $x$ converge to $x^{*}$ as long as the evolving population is monomorphic, then respond to the disruptive selection at $x^{*}$ by creating a dimorphism of trait values around $x^{*}$, and finally cause the divergence of the two stably coexisting branches away from $x^{*}$.

When considering processes of evolutionary branching in sexual populations, selection for reproductive isolation comes into play. As lineage splits are adaptive at evolutionary branching points, in the sense of freeing populations from being stuck at fitness minima, the evolution of premating isolation is favored under such circumstances. Any evolutionarily attainable or already existing mechanism of assortative mating can be recruited by selection to overcome the forces of recombination that otherwise prevent sexual populations from splitting up (e.g., Udovic 1980; Felsenstein 1981). Since there exist a plethora of such mechanisms for assortativeness (based on size, color, pattern, acoustic signals, mating behavior, mating grounds, mating season, 
the morphology of genital organs etc.), and since only one out of these many mechanisms is needed to take effect, it would indeed be surprising if many natural populations would remain stuck at fitness minima for very long (Geritz et al. 2004). Models for the evolutionary branching of sexual populations corroborate that expectation (e.g., Dieckmann and Doebeli 1999; Doebeli and Dieckmann 2000, 2003, 2005; Geritz and Kisdi 2000; Doebeli et al. 2005).

Processes of adaptive speciation (Dieckmann et al. 2004), resulting from the frequency-dependent mechanisms described above, are very different from those stipulated by the standard model of allopatric speciation through geographical isolation (Mayr 1963, 1982), which have dominated speciation research for decades. Closely related to adaptive speciation are models of sympatric speciation (e.g., Maynard Smith 1966; Johnson et al. 1996), of competitive speciation (Rosenzweig 1978), and of ecological speciation (Schluter 2000), which all point in the same direction: patterns of species diversity are not only shaped by exogenous processes of geographical isolation and immigration, which can be more or less arbitrary, but can instead by driven by endogenous processes of selection and evolution, which are bound to imbue such patterns with a stronger deterministic component.

In conjunction with mounting empirical evidence that rates of race formation and sympatric speciation are potentially quite high, at least under certain conditions (e.g., Bush 1969; Meyer 1993; Schliewen et al. 1994), these considerations suggest that understanding processes and patterns of community formation will crucially benefit from notions developed in the context of adaptive speciation.

\subsection{Selection-driven decreases in species numbers}

Frequency-dependent selection and density-dependent selection are also crucial for understanding how selection pressures can decrease the number of species within an ecological community:

- First, in evolutionary game theory - including all evolutionary models based on matrix games or on the replicator equation - a population's density is not usually part of the model, which describes only the frequencies of different types. Without enhancements, these types of model therefore cannot account for any density-dependent selection pressures, or capture selection-driven extinctions during which a population's density drops to zero.

- Second, in optimization approaches of evolution, a constant fitness landscape governs the course and outcome of evolution, and, accordingly, frequency-dependent selection is absent. Again, the density of the evolving population is usually not part of the model. Even when it is, selectiondriven extinctions cannot occur, as no acceptable constant fitness function will be maximized when a population goes extinct. 
These two limitations explain why, until relatively recently, population extinctions caused by natural selection were rarely modeled. In particular, landmarks of evolutionary theory are based on notions of optimizing selection: this includes Fisher's so-called fundamental theorem of natural selection (Fisher 1930) and Wright's notion of hill climbing on fitness landscapes (Wright 1932, 1967). Also Levins's seminal fitness-set approach to the study of constrained bivariate evolution (Levins 1962, 1968) is based on the assumption that, within a set of feasible phenotypes defined by a trade-off, evolution will maximize a population's fitness. Even the advent of evolutionary game theory (Maynard Smith 1982), with its conceptually most valuable refocusing of attention towards frequency-dependent selection, did not help as such, since, for the sake of simplicity, population densities were usually removed from consideration in such models (for an alternative approach to game dynamics aimed at including densities, see Cressman 1990).

And yet the potential of adaptations to cause the collapse of populations was recognized early on. Haldane (1932) provided a verbal example by considering overtopping growth in plants. Taller trees get more sunlight while casting shade onto their neighbors. As selection thus causes the average tree height to increase, fecundity and carrying capacity decline because more of the tree's energy budget is diverted from seed production to wood production, and the age at maturation increases. Arborescent growth as an evolutionary response to selection for competitive ability can therefore cause the decline of a population's abundance as well as of its intrinsic growth rate, potentially resulting in population extinction. The phenomenon of selection-driven extinction is closely related to Hardin's (1968) tragedy of the commons. In both cases, strategies or traits that benefit the selfish interests of individuals, and that are therefore bound to invade, undermine the overall interests of the evolving population as a whole once these strategies or traits have become common. Such a disconnect between individual interest and population interest can only occur under frequency-dependent selection - under optimizing selection, the two are equivalent. It is thus clear that frequency-dependent selection and density-dependent selection are both necessary for capturing the potential of adaptive evolution in a single species to cause its own extinction.

Processes of selection-driven extinction can come in several forms:

- First, evolutionary suicide (Ferrière 2000) is defined as a trait substitution sequence driven by mutation and selection taking a population toward and across a boundary in the population's trait space beyond which the population cannot persist. Once the population's trait values have evolved close enough to this boundary, mutants can invade that are viable as long as the current resident trait value abounds, but that are not viable on their own. When these mutants start to invade the resident population, they initially grow in number. However, once they have become sufficiently abundant, concomitantly reducing the former resident's density, the mutants bring about their own extinction. Webb (2003) refers to such processes of evolutionary suicide as Darwinian extinction. 
- Second, adaptation may cause population size to decline gradually through perpetual selection-driven deterioration. Sooner or later, demographic and environmental stochasticity will then cause population extinction. This phenomenon has been dubbed runaway evolution to self-extinction by Matsuda and Abrams (1994a).

- Third, the population collapse abruptly brought about by an invading mutant phenotype may not directly lead to population extinction but only to a substantial reduction in population size (Dercole et al. 2002). Such a collapse will then render the population more susceptible to extinction by stochastic causes and may thus indirectly be responsible for its extinction.

For univariate traits, the normal form for the invasion fitness of mutants with trait values $x^{\prime}$ in resident populations with trait values $x$ that are close to a critical trait value $x^{*}=0$ at which evolutionary suicide occurs is simply given by

$$
f\left(x^{\prime}, x\right)=x^{\prime}-x,
$$

with the corresponding equilibrium abundance

$$
\bar{n}(x)= \begin{cases}1+c x^{2} & x \leq 0 \\ 0 & x>0\end{cases}
$$

with $c>0$. From this we can see that the selection pressure at $x^{*}$ is positive, $g\left(x^{*}\right)=1>0$, so that trait substitutions in $x$ converge to $x^{*}$, where the evolving population's equilibrium abundance abruptly drops from 1 to 0 .

The occurrence here of a discontinuous transition to extinction is not accidental. As has been explained by Gyllenberg and Parvinen (2001), Gyllenberg et al. (2002), Webb (2003), and Dieckmann and Ferrière (2004), such a catastrophic bifurcation is a strict prerequisite for evolutionary suicide. The reason is that selection pressures at trait values at which a continuous transition to extinction occurs (e.g., through a transcritical bifurcation) always point in the trait direction that increases population size: evolution towards extinction is then impossible. Allee effects, by contrast, provide standard ecological mechanisms for discontinuous transitions to extinction.

The potential ubiquity of selection-driven extinctions is underscored by numerous examples based on the evolutionary dynamics of many different traits, including competitive ability (Matsuda and Abrams 1994a; Gyllenberg and Parvinen 2001; Dercole et al. 2002), anti-predator behavior (Matsuda and Abrams 1994b), sexual traits (Kirkpatrick 1996; Kokko and Brooks 2003), dispersal rates (Gyllenberg et al. 2002), mutualism rates (Ferrière et al. 2002), cannibalistic traits (Dercole and Rinaldi 2002), maturation reaction norms (Ernande et al. 2002), levels of altruism (Le Galliard et al. 2003), and selfing rates (Cheptou 2004). Dieckmann and Ferrière (2004) showed, by examining ecologically explicit multi-locus models, that selection-driven extinction robustly occurs also under sexual inheritance. Relevant overviews of 
the mathematical and ecological underpinnings of selection-driven extinction have been provided by Webb (2003), Dieckmann and Ferrière (2004), Rankin and López-Sepulcre (2005), and Parvinen (2006).

Also coevolutionary dynamics can cause extinctions. An early treatment, which still excluded the effects of intraspecific frequency-dependent selection, was provided by Roughgarden $(1979,1983)$. This limitation has been overcome in modern models of coevolutionary dynamics based, for example, on the canonical equation of adaptive dynamics (e.g., Dieckmann et al. 1995, Dieckmann and Law 1996; Law et al. 1997). Also in this multi-species context it is important to distinguish between continuous and discontinuous transitions to extinction. As has been explained above, evolutionary suicide cannot contribute to an evolutionarily driven continuous transition to extinction. Moreover, such continuous extinctions cause mutation-limited phenotypic evolution in the dwindling species to grind to a halt, since fewer and fewer individuals are around to give birth to the mutant phenotypes that fuel the adaptive process. This stagnation renders the threatened species increasingly defenseless by depriving it of the ability to counteract the injurious evolution of its partner through suitable adaptation of its own. For these two reasons, continuous evolutionary extinctions are driven solely by adaptations in the coevolving partners. By contrast, when a transition to extinction is discontinuous, processes of evolutionary suicide and of coevolutionary forcing may conspire to oust a species from the evolving community.

\subsection{First example of community evolution: monomorphic and deterministic}

Simple community modules comprising two, three, or four interacting species have often been used for investigating how trophic interactions organize simple communities. These studies have laid the foundations for theories (i) of competition, including the $R^{*}$ rule (Tilman 1982), (ii) of predation within the context of exploitative ecosystems, including work on trophic cascades (Oksanen et al. 1981; Oksanen and Oksanen 2000), and (iii) of omnivory, including research on intraguild predation (Holt and Polis 1997; Diehl and Feissel 2000; Mylius et al. 2001; HilleRisLambers and Dieckmann 2003). All these studies, however, did not account for the potential of evolutionary changes in the ecological interactions between the considered species. Overcoming this restriction is important as patterns of species interactions encountered in nature ought to be interpreted in light of not only ecological stability but also of evolutionary stability.

Here we take a step in this direction by investigating the evolution of feeding preferences within a simple community module. In particular, we examine evolutionary dynamics in simple food webs comprising a basal resource and two antagonistic consumer species, where each consumer is capable of feeding on the resource, on its antagonist, or on a combination of both 
(HilleRisLambers and Dieckmann 2003). The relative investments into resource or antagonist feeding characterize the consumers' feeding preferences and can evolve subject to a trade-off. In this way, all of the classic threespecies community modules - including linear food chains, two consumers sharing a resource, omnivory on the part of one consumer, and mutual intraguild predation between two consumers - can be realized in the model. By examining how feeding preferences - and thus the trophic linkages between species - evolve, we can chart the possible evolutionary pathways connecting all these classic community modules (HilleRisLambers and Dieckmann, submitted). Since density- and frequency-dependent selection pressures are important for addressing these questions, and since it is desirable to derive the considered evolutionary dynamics from the underlying population dynamics, models of adaptive dynamics provide a useful framework for this kind of analysis.

The abundances $n_{C}$ and $n_{D}$ of the two antagonistic consumers change according to Lotka-Volterra dynamics, assuming intrinsic mortalities and linear functional responses. The basal energetic input is provided by a dynamic resource, whose abundance $n_{R}$ changes according to semichemostat dynamics and consumer feeding. The community's population dynamics are thus given by

$$
\begin{aligned}
& \frac{\mathrm{d}}{\mathrm{d} t} n_{C}=n_{C}\left(e_{C R} a_{C R} n_{R}+e_{C D} a_{C D} n_{D}-a_{D C} n_{D}-d_{C}\right), \\
& \frac{\mathrm{d}}{\mathrm{d} t} n_{D}=n_{D}\left(e_{D R} a_{D R} n_{R}+e_{D C} a_{D C} n_{C}-a_{C D} n_{C}-d_{D}\right), \\
& \frac{\mathrm{d}}{\mathrm{d} t} n_{R}=r_{R}\left(k_{R}-n_{R}\right)-n_{R}\left(a_{C R} n_{C}+a_{D R} n_{D}\right),
\end{aligned}
$$

with attack coefficients $a$, conversion efficiencies $e$, and intrinsic mortality rates $d$. The carrying capacity and intrinsic growth rate of the resource are denoted by $k_{R}$ and $r_{R}$, respectively.

The feeding preferences of the two consumers are affected by a trade-off between the attack coefficients for resource feeding and antagonist feeding,

$$
a_{i R}=a_{\max , i} x_{i}^{s_{i}}, \quad a_{i j}=a_{\max , i}\left(1-x_{i}\right)^{s_{i}},
$$

for $i=C, D$ and $j=D, C$, where, for consumer $i, a_{\max , i}$ is the maximal attack coefficient, $s_{i}$ is the trade-off strength, and the adaptive trait $0 \leq x_{i} \leq 1$ determines the feeding preference, measured as the relative investment into resource feeding. Intermediate feeding strategies, $0<x_{i}<1$, characterize omnivorous consumers. For $s_{i}>1$, generalist feeding strategies $\left(x_{i} \approx \frac{1}{2}\right)$ are disfavored compared with specialist feeding strategies $\left(x_{i} \approx 0,1\right)$, resulting in specialist advantage. The reverse is true for $s_{i}<1$, which thus corresponds to generalist advantage. On this basis, the canonical equations (Sect. 8.2) for the two adaptive traits $x_{C}$ and $x_{D}$ are given by

$$
\frac{\mathrm{d}}{\mathrm{d} t} x_{i}=\frac{1}{2} \mu_{i} \sigma_{i}^{2} \bar{n}_{i} a_{\max , i} s_{i}\left[e_{i R} x_{i}^{s_{i}-1} \bar{n}_{R}-e_{i j}\left(1-x_{i}\right)^{s_{i}-1} \bar{n}_{j}\right],
$$


for $i=C, D$ and $j=D, C$, where equilibrium abundances are denoted by $\bar{n}$, and $\mu_{i}$ and $\sigma_{i}^{2}$ are the mutation probability and variance in consumer $i$.

Coevolutionary dynamics unfold within the constraints of ecological coexistence. HilleRisLambers and Dieckmann (2003) found that, in the model specified above, regions of coexistence open up around linear three-species food chains, $\left(x_{C}, x_{D}\right)=(0,1),(1,0)$, where one consumer is a better antagonist feeder, while the other consumer is a better resource feeder. When the trade-off strengths $s_{C}$ and $s_{D}$ are varied together, $s_{C}=s_{D}$, two extreme scenarios can be distinguished:

- At strong specialist advantage, linear three-species food chains are evolutionarily stable (in the sense of the corresponding trait combinations being asymptotically stable under the adaptive dynamics described by the two simultaneous canonical equations for $x_{C}$ and $x_{D}$ ). Under these conditions, selection simplifies community structure by causing the evolution of neighboring trait values towards $\left(x_{C}, x_{D}\right)=(0,1),(1,0)$. This means that the better resource feeder will invest even more into resource feeding, while the better antagonist feeder will invest even more into antagonist feeding, until the evolving three-species food chain has become strictly linear.

- At strong generalist advantage, trait combinations ensuring ecological coexistence are severely limited (HilleRisLambers and Dieckmann 2003). Under these conditions, linear three-species food chains become evolutionarily unstable, and both the better resource feeder and the better antagonist feeder evolve towards generalist strategies, which ultimately results in the exclusion of the former by the latter. Also here the end result is a simplified community structure, in this case given by a simple two-species food chain.

At intermediate trade-off strengths, ecologically feasible communities evolve towards linear two- or three-species food chains, largely depending on the initial feeding preference of the better antagonist feeder.

It must be expected that the trade-offs constraining the attack coefficients of consumer species are not identical, $s_{C} \neq s_{D}$. Considering intermediate trade-off strengths, we find that if the better antagonist feeder is more constrained at generalist feeding strategies than the better resource feeder, linear food chains are evolutionarily unstable, and evolutionarily stable food webs exhibiting more complex trophic interactions may be realized. Fig. 8.3a shows such a coevolutionary attractor with $\left(x_{C}, x_{D}\right) \neq(0,1),(1,0)$. Fig. 8.3a also shows that different coevolutionary attractors may coexist. Depending on the initial food web configuration, coevolution leads to one of the three outcomes depicted in Fig. 8.3b: (i) coexistence between two omnivores, (ii) coexistence between an omnivore and a pure antagonist feeder, or (iii) evolutionary exclusion of the better resource feeder. Which of these occurs is affected largely by the initial feeding preference of the better antagonist feeder and also by the relative scaling of the evolutionary rates in the two 
(a)

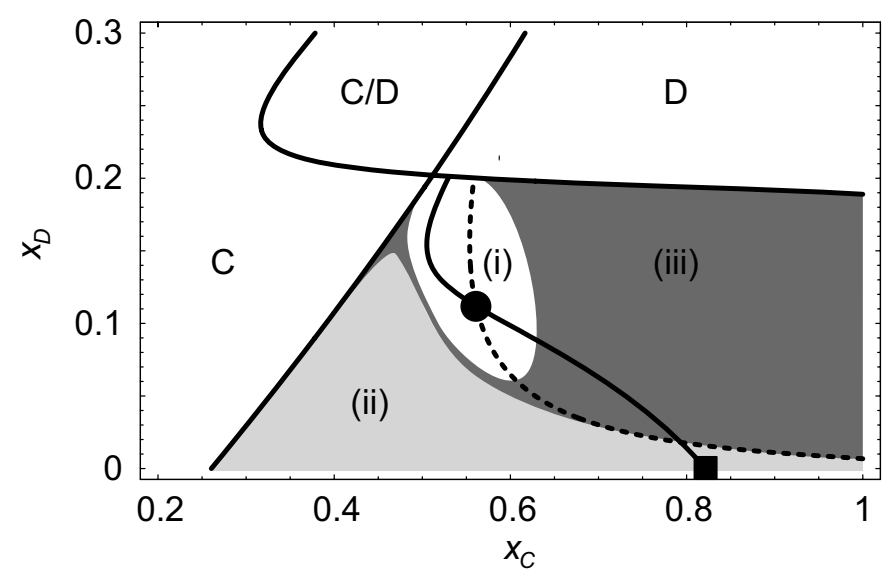

(b)

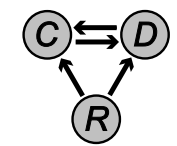

(i)

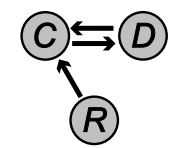

(ii)

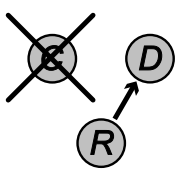

Fig. 8.3. Evolution of community structure in first example. Traits $x_{C}$ and $x_{D}$ measure the degree to which consumers $\mathrm{C}$ and $\mathrm{D}$ invest into feeding on the resource $\mathrm{R}$, as opposed to feeding on each other. For $x_{C}>x_{D}$, C is the better resource feeder, while $\mathrm{D}$ is the better antagonist feeder. In panel (a), the evolutionary isoclines of $x_{C}$ and $x_{D}$ are depicted by continuous and dashed curves, respectively. Regions in panel (a) indicate different potentials for coexistence and coevolution. Region C: $\mathrm{C}$ and $\mathrm{R}$ can coexist, while $\mathrm{D}$ goes extinct. Region $\mathrm{D}: \mathrm{D}$ and $\mathrm{R}$ can coexist, while $\mathrm{C}$ goes extinct. Region $\mathrm{C} / \mathrm{D}$ : ecological bistability between coexistence of $\mathrm{R}$ with either C or D. Regions (i), (ii), and (iii): C, D, and R can coexist, so that C and $\mathrm{D}$ can coevolve. The community structures resulting from these coevolutionary dynamics then depend on the initial conditions for $\left(x_{C}, x_{D}\right)$ and are shown in panel (b). Region (i): Coevolution towards attractor depicted by filled circle, corresponding to omnivorous mutual intraguild predation. Region (ii): Coevolution towards attractor depicted by filled square, corresponding to omnivory on the part of just one consumer. Region (iii): Coevolution towards Region D, corresponding to the exclusion of consumer C. Parameters: $s_{C}=0.82, s_{D}=1.5, a_{\max , C}=a_{\max , D}=0.4$, $e_{C R}=e_{D R}=0.2, e_{C D}=e_{D C}=0.8, d_{C}=d_{D}=0.05, r_{R}=0.2, k_{R}=100$, $\mu_{C} \sigma_{C}^{2} / \mu_{D} \sigma_{D}^{2}=5$.

consumers, measured by $\mu_{C} \sigma_{C}^{2} / \mu_{D} \sigma_{D}^{2}$. Specifically, the basin of attraction for outcome (iii) increases when the better antagonist feeder evolves faster than the better resource feeder. It is also possible that communities of type (i) exhibit cyclical fluctuations in the feeding preferences $x_{C}$ and $x_{D}$, akin to those found by Dieckmann et al. (1995) for predator-prey coevolution and by Law et al. (1997) for coevolution under asymmetric competition. These evolutionary cycles may come dangerously close to the boundaries of coexistence, so that small environmental perturbations may then lead to a shift from outcome (i) to (iii).

We can summarize the results of the analysis here by concluding that linear three-species food chains are most likely to persist evolutionarily under 
strong specialist advantage, whereas the evolutionary exclusion of consumers is most likely under strong generalist advantage. By contrast, complex trophic interactions in this model are difficult to stabilize evolutionarily. They are most likely to occur in communities in which trade-off strengths are intermediate and the better antagonist feeder experiences a stronger trade-off than the better resource feeder, especially when the latter evolves faster than the former.

\subsection{Second example of community evolution: oligomorphic and stochastic}

Some existing models of food web evolution incorporate realistic population dynamics, but at the same time rely on interactions mediated by highdimensional traits that lack clear and direct ecological interpretations (e.g., Caldarelli et al. 1998; Drossel al. 2001). By contrast, a model by Brännström et al. (in preparation), described below, builds on previous foundational work by Loueille and Loreau (2005) and accordingly is based on body size as an evolving trait of high physiological and ecological relevance.

The considered community comprises one autotrophic and $N$ heterotrophic species evolving through mutation-limited phenotypic adaptation. Each species $i$ possesses a trait value $x_{i}$ determining its body size on a logarithmic scale. From these body sizes, species-specific properties such as energy requirements, competitive interactions, and attack coefficients are determined. The community's demographic processes follow Lotka-Volterra dynamics, with the dynamics of the non-evolving autotrophic species $i=0$ given by

$$
\frac{\mathrm{d}}{\mathrm{d} t} n_{0}=n_{0}\left[b_{0}-n_{0} / k_{0}-\sum_{j=1}^{N} \exp \left(x_{j}-x_{0}\right) F\left(x_{j}-x_{0}\right) n_{j}\right]
$$

and the per capita birth and death rates, respectively, of the heterotrophic species $i=1, \ldots, N$ given by

$$
\begin{aligned}
& b_{i}(x, n)=e \sum_{j=0}^{N} \exp \left(x_{j}-x_{i}\right) F\left(x_{i}-x_{j}\right) n_{j} \\
& d_{i}(x, n)=d\left(x_{i}\right)+\sum_{j=1}^{N} F\left(x_{j}-x_{i}\right) n_{j}+\sum_{j=1}^{N} C\left(x_{i}-x_{j}\right) \exp \left(x_{j}\right) n_{j} .
\end{aligned}
$$

The four terms on the two right-hand sides above correspond, in turn, to reproduction, intrinsic mortality, mortality from predation, and mortality from interference competition:

- Energy inflow from foraging results in reproduction as described by the first term. The rate at which new individuals enter the focal species 
through birth thus depends on the abundance of available prey, on the relative difference in size between predator and prey, and on a predator's ability to attack a prey. The latter is characterized by a shifted Gaussian function $F$ of the relative size difference, with $F$ being referred to as the foraging kernel. The degree to which energy is lost as prey biomass is converted into offspring is measured by the trophic efficiency $e$.

- The intrinsic mortality rate in the second term is assumed to decrease with body size according to a power law resulting in body-size-dependent generation times consistent with empirical observations (e.g., Peters 1983).

- Losses resulting from predation are captured by the third term, which immediately follows from the considerations concerning the foraging kernel.

- Interference competition between individuals is described by the fourth term. The increase in mortality caused by interference from other individuals depends on their biomass and on the relative size difference. This is characterized by a Gaussian function $C$, centered at zero and referred to as the competition kernel. Accordingly, two individuals that greatly differ in size will compete much less than two individuals that have similar sizes. The exponential term ensures that smaller individuals are affected more by interference competition.

The evolutionary dynamics of this community are modeled under the assumption that mutations are rare, so that a new mutant will either successfully invade the resident community or be extinct by the time the next successful mutation occurs. We can then employ an oligomorphic extension of the evolutionary random walk model described in Sect. 8.2. Mutations occur at a rate proportional to the total birth rate of the corresponding resident species, and mutant trait values are assumed to be normally distributed around those of their parent. Whether or not a mutant morph can invade the resident community will depend on its invasion fitness, with the success probabilities of potentially invading mutants given in Sect. 8.2. When a successful invasion occurs, its community-level consequences can be determined from the LotkaVolterra dynamics specified above. However, since the underlying time integration is time-consuming, an approximate, but in practice accurate, algorithm is used, known as the oligomorphic stochastic model (Ito and Dieckmann, unpublished). The steps in this algorithm aim at inferring the structure of the post-invasion community without time integration whenever possible. Simulations of the evolutionary process end when the community-level probability of successful invasion falls below a prescribed threshold.

Fig. 8.4a shows how the interplay between mutation and selection gradually leads from a single ancestral species to a community of seven heterotrophic species, through a process of sequential evolutionary branching. The structure of the resulting food web is depicted in Fig. 8.4b.

To isolate and determine the factors governing diversity, two complementary approaches were used. First, the asymptotic number of species was evaluated numerically, as described above. Second, the strengths of disruptive 
(a)

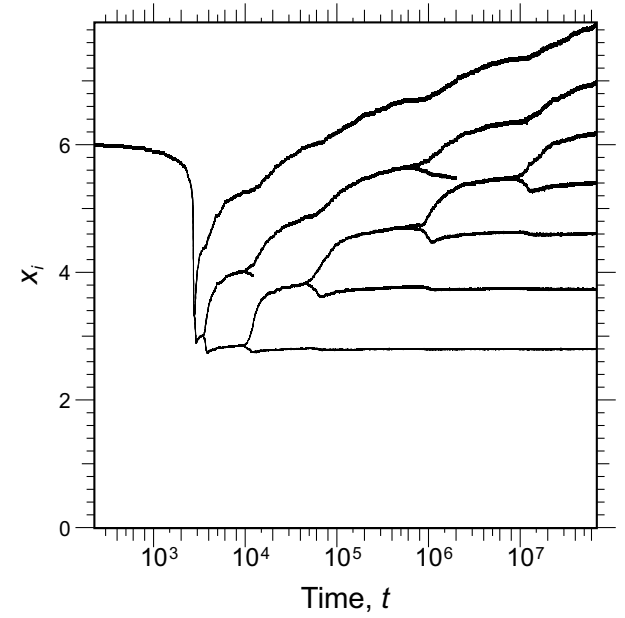

(b)

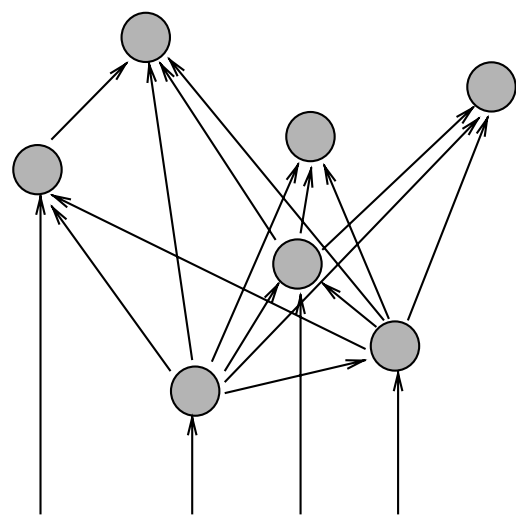

Fig. 8.4. Evolution of community structure in second example. Panel (a) shows the temporal development of community structure through recurrent evolutionary branching, utilizing a logarithmic time scale. Panel (b) depicts the resulting community structure. Each species is represented by a circle, with its vertical position given by its trophic level. Circles are connected by arrows, from prey to predator, where the energy flow between the two corresponding species account for more than $10 \%$ of the total energy inflow to the recipient species. Arrows connecting to the bottom indicate consumption of the autotrophic species (or basal resource, which is not displayed). Parameters: $x_{0}=1, k_{0}=100, b_{0}=1, e=0.3, d\left(x_{i}\right)=d_{0} \exp \left(-q x_{i}\right)$ with $d_{0}=0.2$ and $q=0.75 ; F$ is a lognormal function with mean 3 , standard deviation 1.5, and amplitude 2.5; $C$ is a lognormal function with mean 0 , standard deviation 0.6 , and amplitude 0.0025 .

selection at the first and second branching points were determined as a function of model parameters. This enabled analytical insights into which parameters are important for the initial stages of food web evolution. Interference competition and metabolic scaling (in the form of reduced mortality at larger body size) proved to be critical components in this regard. The former promotes evolutionary branching and is a prerequisite for diversity to develop, while the latter offsets the advantage that smaller species enjoy in terms of increased encounter rates per unit of biomass. In simulations in which either interference competition or metabolic scaling were absent, evolution did not lead to communities with more than just one or two species.

It proved useful to group parameters according to their role in the model, with energy parameters directly affecting the energy flow, foraging parameters determining the shape of the foraging kernel, and competition parameters governing the interference competition between individuals of similar size. With this grouping and terminology in place, it turned out that the initial 
stages of food web evolution primarily depend on the energy and competition parameters. While these same parameters were naturally also important for the asymptotically evolving diversity, their role there was largely overshadowed by the foraging parameters. The fact that some parameters are mainly important in the early stages of community evolution while others become crucial only during the later stages shows that an analysis that stopped prematurely after investigating only the first or second incidence of evolutionary branching would be insufficient for determining which mechanisms and parameters affect the longer-term structuring of ecological communities.

\subsection{Third example of community evolution: polymorphic and deterministic}

Explaining the evolutionary origin and history of food webs through sequential adaptive diversification is a challenge that has as yet been tackled by few evolutionary models. It is therefore interesting to explore to what extent the coevolution of predator-prey interactions underlying trophic community structures can induce recurrent evolutionary branching.

In nature the ecological dynamics of phenotypes engaged in trophic interactions depend on how the considered individuals perform in their roles as predator on the one hand and as prey on the other. Both of these components must be expected to evolve. Ito and Ikegami $(2003,2006)$ therefore considered bivariate adaptive traits $x=\left(x_{r}, x_{u}\right)$, with the first trait component $x_{r}$ determining how an individual is exposed as a resource (strategy as prey) and the second trait component $x_{u}$ determining how the individual is utilizing such resources (strategy as predator). Resources may have many relevant phenotypic properties - including body size, toxicity, proportion of protective tissue, ability to hide, running speed etc. - which jointly can be described by a vector $z$. The contribution an individual with resource trait $x_{r}$ makes to the density in this potentially multivariate resource space is denoted by $p_{r}\left(x_{r}, z\right)$, and analogously the utilization spectrum of an individual with utilization trait $x_{u}$ is $p_{u}\left(x_{u}, z\right)$. Given a phenotypic distribution $p(x)$, the distribution of resource properties is thus $P_{r}(z)=\iint p\left(x_{r}, x_{u}\right) d x_{u} p_{r}\left(x_{r}, z\right) \mathrm{d} x_{r}+S(z)$, where $S$ accounts for sources of resource supply from outside the modeled population. Likewise, the population's utilization spectrum is $P_{u}(z) x=x \iint p\left(x_{r}, x_{u}\right) d x_{r} p_{u}\left(x_{u}, z\right) \mathrm{d} x_{u}$. Ito and Ikegami $(2003,2006)$ then considered the following ecological and evolutionary dynamics,

$$
\begin{aligned}
\frac{\mathrm{d}}{\mathrm{d} t} p(x)= & \left(e \int F(z) p_{u}\left(x_{u}, z\right) \mathrm{d} z-\int F(z) \frac{P_{u}(z)}{P_{r}(z)} p_{r}\left(x_{r}, z\right) \mathrm{d} z-d\right) p(x) \\
& +\frac{1}{2} \mu \sigma^{2} * \frac{\partial^{2}}{\partial x^{2}} p(x),
\end{aligned}
$$


where $e$ measures trophic conversion efficiency and $d$ is the intrinsic death rate. The function $F(z)=a P_{r}(z) /\left(1+P_{r}(z) / P_{1 / 2}\right)$ is a Holling type II functional response, with maximum $a$ and half-saturation constant $P_{1 / 2}$. As explained in Sect. 8.2 and in the Appendix, the population-level effect of frequent mutations can be approximated by a diffusion term with diffusion coefficient matrix $\frac{1}{2} \mu \sigma^{2}$ (to avoid dynamical artifacts, values of $p(x)$ are reset to zero after falling below a very low cutoff threshold).

(a)

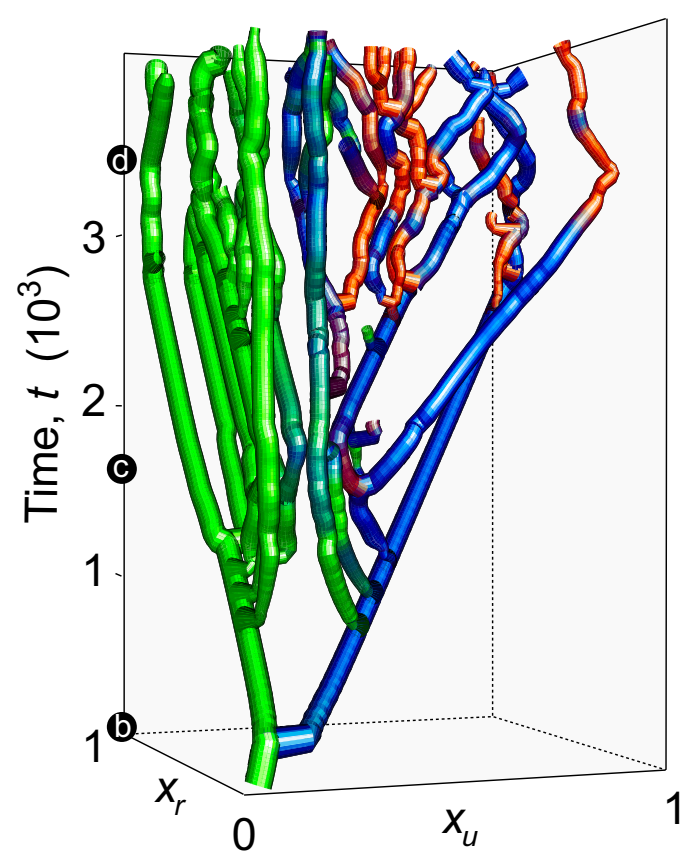

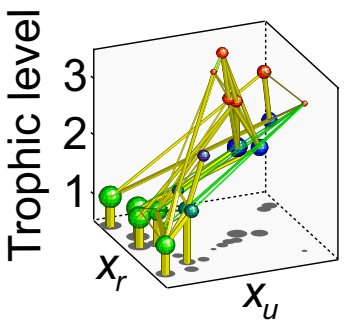

(d)

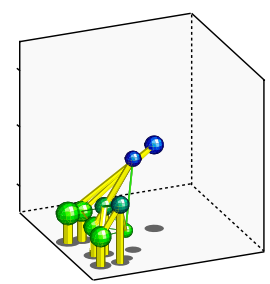

(c)

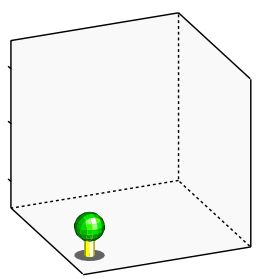

Fig. 8.5. Evolution of community structure in third example. When two trait components for an individual's strategy as prey, $x_{r}$, and for its strategy as predator, $x_{u}$, evolve under selection pressures resulting from predator-prey interactions, complex food webs can emerge through recurrent evolutionary branching. Panel (a) shows the temporal development of community structure, with the widths of tubes reflecting the densities of phenotypic clusters. Panels (b) to (d) show the evolving food web at three different moments in time. Spheres represent phenotypic clusters, with diameters reflecting the corresponding densities. On the bottom planes, the shadows of these spheres show the distribution $p(x)$. Tubes represent trophic links, with diameters reflecting the corresponding interaction strengths. Tubes connecting to the bottom planes indicate consumption of the external supply of resources (which is assigned trophic level 0 ). The resultant trophic levels of phenotypic clusters are shown along the vertical axes in (b) to (d). Parameters: $e=0.1, d=1, a=20$, $P_{1 / 2}=17, \frac{1}{2} \mu \sigma^{2}=\left(\left(3 \cdot 10^{-2}, 0\right),\left(0,10^{-3}\right)\right), S_{0}=200, \sigma_{S}=0.08$. 
For the sake of simplicity, here we assume a one-dimensional resource space, strictly localized functions $p_{r}\left(x_{r}, z\right)=\delta\left(z-x_{r}\right)$ and $p_{u}\left(x_{u}, z\right)=$ $\delta\left(z-x_{u}\right)$, where $\delta$ denotes Dirac's delta function, a normally distributed source of external resources, $S(z)=S_{0} N_{0, \sigma_{S}^{2}}(z)$, and traits $x_{r}$ and $x_{u}$ confined to the unit interval. Within a wide range of parameter values, the dynamics of initially unimodal phenotypic distributions $p(x)$ then comprises phases of directional evolution and evolutionary branching. Phenotypic clusters with few prey and many predators go extinct, while phenotypic clusters with many prey and few predators rapidly increase in density and subsequently split through evolutionary branching. Since branching in $x_{r}$ often induces branching in $x_{u}$, and vice versa, the branching sequences resulting from this positive feedback bring about a richly structured food web. Large food webs are maintained through a dynamic balance between selection-driven branching and extinction.

Implementation of sexual reproduction, akin to the model by Drossel and McKane (2000), does not change these dynamics qualitatively (apart from the fact that phenotypic clusters become reproductively isolated). Giving $p_{r}$ and $p_{u}$ a certain width, by assuming Gaussian functions instead of delta functions, also does not qualitatively affect evolutionary outcomes. Finally, interference competition among predators can be considered by using $F(z)=a P_{r}(z) /\left(P_{u}(z)+P_{r}(z) / P_{1 / 2}\right)$, which gives rise to a ratio-dependent functional response (Arditi and Ginzburg 1989) and facilitates the evolutionary origin and maintenance of complex food webs, as illustrated in Fig. 8.5.

\subsection{Fourth example of community evolution: polymorphic and stochastic}

The examples presented so far may create the impression that trophic interactions were a necessary prerequisite for the evolutionary origin and maintenance of complex community structures. This is clearly not the case. Purely competitive interactions have long been shown to ensure the maintenance of large species numbers, with early work on the species packing problem dating back to MacArthur and Levins (1967), Vandermeer (1970), May (1973), and Roughgarden (1974).

To illustrate and underscore the potential of purely competitive interactions to bring about and structure multi-species communities through evolutionary dynamics including adaptive radiations, we consider adaptations under asymmetric competition. Specifically, we assume that interactions between individuals are affected by a univariate quantitative trait $x$, of which we may think, for example, as representing stem height in plants or adult body size in animals. In either case, individuals with a small trait value will suffer a lot from competition against individuals with a large trait value, while the reverse effects will often be negligible. And if individuals are too far apart in their trait values, so as to occupy essentially different ecological 
niches, they will hardly interact at all. These qualitative dependencies are captured by the function

$$
C\left(x-x^{\prime}\right)=\exp \left(\frac{1}{2} \sigma_{C}^{2} \beta^{2}\right) \exp \left(-\frac{\left(x-x^{\prime}+\sigma_{C}^{2} \beta\right)^{2}}{2 \sigma_{C}^{2}}\right),
$$

which has been used to describe the strength of competition exerted by an individual with trait value $x^{\prime}$ on an individual with trait value $x$ (Rummel and Roughgarden 1985; Taper and Case 1992). Here $\beta=0$ corresponds to symmetric competition, while $\beta>0$ causes asymmetric competition favoring larger trait values. We also assume that trait values differ in their intrinsic carrying capacity,

$$
K(x)=K_{0} \exp \left(-\frac{1}{2}\left(x-x_{0}\right)^{2} / \sigma_{K}^{2}\right),
$$

which, by itself, causes stabilizing selection towards $x=x_{0}$. On this basis, we can specify the per capita birth and death rates of individuals with trait values $x$ in a community with phenotypic density $p$, $b(x, p)=b_{0}, \quad d(x, p)=\frac{1}{K(x)} \int C\left(x-x^{\prime}\right) p\left(x^{\prime}\right) \mathrm{d} x^{\prime}=\frac{1}{K(x)} \sum_{k=1}^{n} C\left(x-x_{k}\right)$, resulting in simple population dynamics of Lotka-Volterra type.

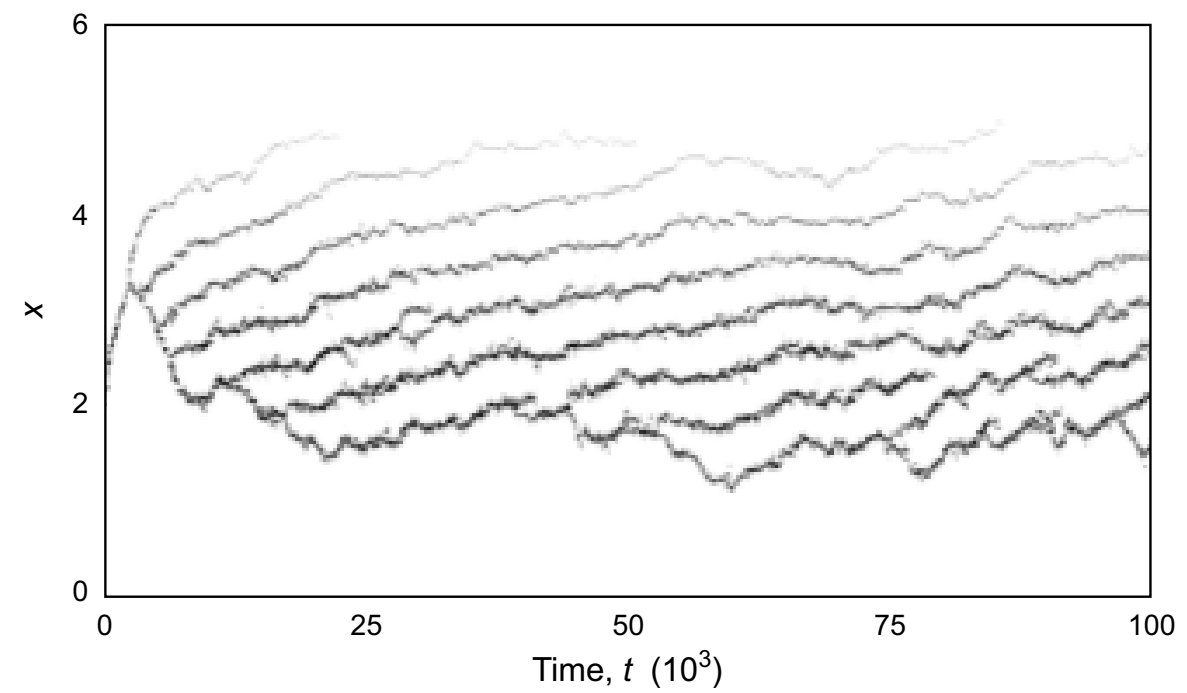

Fig. 8.6. Evolution of community structure in fourth example. When a trait governing asymmetric competition evolves, selection-driven increases and decreases in morph number are embedded into a macroevolutionary pattern of perpetual laminar flow of morphs towards larger trait values. The individual-based dynamics shown involved more than 420,000,000 explicitly simulated birth and death events. Parameters: $b_{0}=1, K_{0}=1000, x_{0}=2, \sigma_{K}=1, \sigma_{C}=0.3, \beta=2, \mu=0.005$, $\sigma=0.025$. 
The individual-based birth-death-mutation model introduced in Sect. 8.2 is well suited to explore the resultant evolutionary dynamics (Doebeli and Dieckmann 2000). Fig. 8.6 shows a typical realization of this stochastic process. As we can see, directional selection towards larger trait values initially causes convergence to a primary evolutionary branching point. Evolutionary branching subsequently enlarges the number of morphs in the community, until the maximum number resulting from limitations on species packing has been reached. Perpetual coevolutionary change then ensues, through (i) the selection-driven extinction of morphs with large trait values, which run out of carrying capacity, (ii) the laminar and gradual flow of resident morphs towards the larger trait values favored by asymmetric competition, and (iii) the continual replenishment of morphs at low trait values through adaptive radiations triggered by the opening up of ecological opportunities resulting from the first two effects. It is worthwhile to highlight that in this model the incessant coevolutionary turnover is caused entirely by intrinsic or endogenous mechanisms. No environmental forcing needs to be invoked for understanding the systematic trends in the observed macroevolutionary pattern.

\subsection{Summary}

In this chapter we have shown how models of adaptive dynamics provide a variety of flexible tools for studying the evolutionary dynamics of ecological communities. Once demography and mutations have been specified, evolutionary and coevolution processes - including those that increase or decrease the number of species in the community - can be analyzed at several mutually illuminating levels of description. While individual-based descriptions of birth, deaths, and mutations provide the finest level of detail, such models are often too computationally intensive and too unwieldy to be comprehensively examined. It is then helpful to have available other classes of models that provide tried and tested approximations. Depending on the features of the evolving community and the nature of the addressed research questions, evolutionary random walks, gradient-ascent models, or reaction-diffusion models may alternatively be best suited for systematically investigating evolving community structures.

Until relatively recently, community models have focused either on the ecological dynamics of large communities or on the evolutionary dynamics of small communities. Now the time seems ripe to bring together these two previously independent strands of inquiry in a new, more ambitious synthesis. Even though it thus has already become clear that a rich diversity of ecological mechanisms can drive the persistent diversification of ecologically relevant adaptive traits, and thus of ecological community structure, much research remains to be done in this area. The eventual goal will be to arrive at a systematic understanding of the ways through which processes of interaction, immigration, and adaptation can work together to generate the 
rich, but at the same time not arbitrary, community structures observed in nature.

Theoretical models of community evolution are revealing the stunning capacity of ecological interactions, in conjunction with the selection pressures thus engendered, to result in the emergence of non-random community patterns. It thus seems safe to conclude that neither of the old Clementsian or Gleasonian notions - viewing ecological communities as either organismically or externally structured - can do justice to the subtle interplay of endogenous and exogenous demographic and evolutionary pressures unfolding in real communities. Fueled by the mutual shaping and reshaping of ecological niches caused by community evolution, natural community structures appear to occupy a highly complex middle ground.

\subsection{Appendix: Specification and derivation of adaptive dynamics models}

This appendix provides salient mathematical details on how the four models of adaptive dynamics are defined and derived.

Polymorphic Stochastic Model. We start from an individual-based description of the ecology of an evolving multi-species community (Dieckmann 1994; Dieckmann et al. 1995). The number of species in the considered community is $N$. The phenotypic distribution $p_{i}$ of a population of $n_{i}$ individuals in species $i$ is given by $p_{i}=\sum_{k=1}^{n_{i}} \delta_{x_{i k}}$, where $x_{i k}$ are the trait values of individual $k$ in species $i$, and $\delta_{x_{i k}}$ denotes the Dirac delta function peaked at $x_{i k}, \delta_{x_{i k}}\left(x_{i}\right)=\delta\left(x_{i}-x_{i k}\right)$. As a reminder we mention that Dirac's delta function is defined algebraically through its so-called sifting property, $\int F\left(x_{i}\right) \delta\left(x_{i}-x_{0}\right) \mathrm{d} x_{i}=F\left(x_{0}\right)$ for any continuous function $F$. This implies $p_{i}\left(x_{i}\right)=0$ unless $x_{i}$ is represented in species $i$. We can thus think of $p_{i}\left(x_{i}\right)$ as a density distribution in the trait space of species $i$, with one peak positioned at the trait value of each individual in that species. Since $\int \delta_{x_{i k}}(x) \mathrm{d} x=1$ for any $x_{i k}$, we also have $\int p_{i}\left(x_{i}\right) \mathrm{d} x_{i}=n_{i}$. If $p_{i}\left(x_{i}\right) \neq 0$ for more than one $x_{i}$, the population in species $i$ is called polymorphic, otherwise it is referred to as being monomorphic. The community's phenotypic composition is described by $p=\left(p_{1}, \ldots, p_{N}\right)$.

The birth and death rates of an individual with trait value $x_{i}$ in species $i$ are given by $b_{i}\left(x_{i}, p\right)$ and $d_{i}\left(x_{i}, p\right)$. Each birth by a parent with trait value $x_{i}$ gives rise, with probability $\mu_{i}\left(x_{i}\right)$, to mutant offspring with a trait value $x_{i}^{\prime} \neq x_{i}$, distributed according to $M_{i}\left(x_{i}^{\prime}, x_{i}\right)$, whereas with probability $1-\mu_{i}\left(x_{i}\right)$ trait values are inherited faithfully from parent to offspring. A master equation (e.g., van Kampen 1981) describes the resultant birthdeath-mutation process,

$$
\frac{\mathrm{d}}{\mathrm{d} t} P(p)=\int\left[r\left(p, p^{\prime}\right) P\left(p^{\prime}\right)-r\left(p^{\prime}, p\right) P(p)\right] \mathrm{d} p^{\prime} .
$$


The equation describes changes in the probability $P(p)$ for the evolving community to be in state $p$. This probability increases with transitions from states $p^{\prime} \neq p$ to $p$ (first term) and decreases with transitions away from $p$ (second term). A birth event in species $i$ causes a single Dirac delta function, peaked at the trait value $x_{i}$ of the new individual, to be added to $p_{i}, p \rightarrow p^{\prime}=p+u_{i} \delta_{x_{i}}$, where the elements of the unit vector $u_{i}$ are given by Kronecker delta symbols, $u_{i}=\left(\delta_{1 i}, \ldots, \delta_{N i}\right)$. Analogously, a death event in species $i$ corresponds to subtracting a Dirac delta function from $p, p \rightarrow p^{\prime}=p-u_{i} \delta_{x_{i}}$.

The rate $r\left(p^{\prime}, p\right)$ for the transition $p \rightarrow p^{\prime}$ is thus given by

$r\left(p^{\prime}, p\right)=\sum_{i=1}^{N} \int\left[r_{i}^{+}\left(x_{i}, p\right) \Delta\left(p+u_{i} \delta_{x_{i}}-p^{\prime}\right)+r_{i}^{-}\left(x_{i}, p\right) \Delta\left(p-u_{i} \delta_{x_{i}}-p^{\prime}\right)\right] \mathrm{d} x_{i}$.

Here $\Delta$ denotes the generalized delta function introduced by Dieckmann (1994), which extends the sifting property of Dirac's delta function to function spaces, i.e., $\int F(p) \Delta\left(p-p_{0}\right) \mathrm{d} p=F\left(p_{0}\right)$ for any continuous functional $F$. The terms $\Delta\left(p+u_{i} \delta_{x_{i}}-p^{\prime}\right)$ and $\Delta\left(p-u_{i} \delta_{x_{i}}-p^{\prime}\right)$ thus ensure that the transition rate $r$ vanishes unless $p^{\prime}$ can be reached from $p$ through a birth event (first term) or death event (second term) in species $i$. The death rate $r_{i}^{-}\left(x_{i}, p\right)$ is given by multiplying the per capita death rate $d_{i}\left(x_{i}, p\right)$ with the density $p_{i}\left(x_{i}\right)$ of individuals at that trait value,

$$
r_{i}^{-}\left(x_{i}, p\right)=d_{i}\left(x_{i}, p\right) p_{i}\left(x_{i}\right) .
$$

Similarly, the birth rate $r_{i}^{+}\left(x_{i}, p\right)$ at trait value $x$ is given by

$$
r_{i}^{+}\left(x_{i}, p\right)=\left[1-\mu_{i}\left(x_{i}\right)\right] b_{i}\left(x_{i}, p\right) p_{i}\left(x_{i}\right)+\int \mu_{i}\left(x_{i}^{\prime}\right) b_{i}\left(x_{i}^{\prime}, p\right) p_{i}\left(x_{i}^{\prime}\right) M_{i}\left(x_{i}^{\prime}, x_{i}\right) \mathrm{d} x_{i}^{\prime},
$$

with the first and second terms corresponding to births without and with mutation, respectively. The master equation above, together with its transition rates, describes so-called generalized replicator dynamics (Dieckmann 1994) and offers a generic formal framework for deriving simplified descriptions of individual-based mutation-selection processes.

Monomorphic Stochastic Model. If the time intervals between successfully invading mutations are long enough for evolution to be mutation-limited, $\mu_{i}\left(x_{i}\right) \rightarrow 0$ for all $i$ and $x_{i}$, the evolving populations will remain monomorphic at almost any moment in time (unless and until evolutionary branching occurs). We can then consider trait substitutions resulting from the successful invasion of mutants into monomorphic resident populations that have attained their ecological equilibrium. Denoting trait values and population sizes by $x_{i}$ and $n_{i}$ for the residents in species $i=1, \ldots, N$ and by $x_{j}^{\prime}$ and $n_{j}^{\prime}$ for a mutant in species $j$, we can substitute the density $p=\left(n_{1} \delta_{x_{1}}, \ldots, n_{N} \delta_{x_{N}}\right)+u_{j} n_{j}^{\prime} \delta_{x_{j}^{\prime}}$ into the generalized replicator dynamics defined above to obtain a master equation for the probability $P\left(n, n_{j}^{\prime}\right)$ of jointly observing resident population sizes $n$ and mutant population size $n_{j}^{\prime}$. 
Assuming that the mutant is rare while the residents are sufficiently abundant to be described deterministically, this master equation is equivalent to the joint dynamics

$$
\frac{\mathrm{d}}{\mathrm{d} t} n_{i}=\left[b_{i}\left(x_{i}, p\right)-d_{i}\left(x_{i}, p\right)\right] n_{i}
$$

for the resident populations with $i=1, \ldots, N$ and

$$
\frac{\mathrm{d}}{\mathrm{d} t} P\left(n_{j}^{\prime}\right)=b_{j}\left(x_{j}^{\prime}, p\right) P\left(n_{j}^{\prime}-1\right)+d_{j}\left(x_{j}^{\prime}, p\right) P\left(n_{j}^{\prime}+1\right)
$$

for the mutant population in species $j$, where $p=\left(m_{1} \delta_{x_{1}}, \ldots, m_{N} \delta_{x_{N}}\right)$ and $P\left(m_{j}^{\prime}\right)$ denotes the probability of observing mutant population size $m_{j}^{\prime}$. The rare mutant thus follows a homogeneous and linear birth-death process.

Assuming that the resident community is at its equilibrium, the conditions $b_{i}\left(x_{i}, \bar{p}\right)=d_{i}\left(x_{i}, \bar{p}\right)$ for all species $i=1, \ldots, N$ define $\bar{n}_{i}(x)$ and thus $\bar{p}(x)=\left(\bar{n}_{1}(x) \delta_{x_{1}}, \ldots, \bar{n}_{N}(x) \delta_{x_{N}}\right), \bar{b}_{j}\left(x_{j}^{\prime}, x\right)=b_{j}\left(x_{j}^{\prime}, \bar{p}(x)\right), \bar{d}_{j}\left(x_{j}^{\prime}, x\right)=$ $d_{j}\left(x_{j}^{\prime}, \bar{p}(x)\right)$, and $\bar{f}_{j}\left(x_{j}^{\prime}, x\right)=\bar{b}_{j}\left(x_{j}^{\prime}, x\right)-\bar{d}_{j}\left(x_{j}^{\prime}, x\right)$. When the resident population in species $j$ is small enough to be subject to accidental extinction through demographic stochasticity, $s_{j}\left(x_{j}^{\prime}, x\right)=\left(1-e^{-2 \tilde{f}_{j}\left(x_{j}^{\prime}, x\right)}\right) /(1-$ $\left.e^{-2 \tilde{f}_{j}\left(x_{j}^{\prime}, x\right) \bar{n}_{j}(x)}\right)$ with $\left.\tilde{f}_{j}\left(x_{j}^{\prime}, x\right)=\bar{f}_{j}\left(x_{j}^{\prime}, x\right)\right) /\left[\bar{b}_{j}\left(x_{j}^{\prime}, x\right)+\bar{d}_{j}\left(x_{j}^{\prime}, x\right)\right]$ approximates the probability of a single mutant individual with trait value $x_{i}^{\prime}$ to survive accidental extinction through demographic stochasticity and to go to fixation by replacing the former resident with trait value $x_{i}$ (e.g., Crow and Kimura 1970). When the resident population in species $j$ is large, $\bar{n}_{j}(x) \rightarrow \infty$, this probability converges to the simpler expression $s_{j}\left(x_{j}^{\prime}, x\right)=\max \left(0, \bar{f}_{j}\left(x_{j}^{\prime}, x\right)\right) / \bar{b}_{j}\left(x_{j}^{\prime}, x\right)$ known from branching process theory (e.g., Athreya and Ney 1972).

Once mutants have grown beyond the range of low population sizes in which accidental extinction through demographic stochasticity is still very likely, they are generically bound to go to fixation and thus to replace the former resident, provided that their trait value is sufficiently close to that of the resident, $x_{j}^{\prime} \approx x_{j}$ (Geritz et al. 2002). Hence the transition rate $r\left(x^{\prime}, x\right)$ for the trait substitution $x \rightarrow x^{\prime}$ is given by multiplying (i) the distribution $\mu_{j}\left(x_{j}\right) \bar{b}_{j}\left(x_{j}, x\right) M_{j}\left(x_{j}^{\prime}, x_{j}\right)$ of arrival rates for mutants $x_{j}^{\prime}$ among residents $x$, with (ii) the probability $s_{j}\left(x_{j}^{\prime}, x\right)$ of mutant survival given arrival, and with (iii) the probability 1 of mutant fixation given survival,

$$
r\left(x^{\prime}, x\right)=\sum_{j=1}^{N} \mu_{j}\left(x_{j}\right) \bar{b}_{j}\left(x_{j}, x\right) M_{j}\left(x_{j}^{\prime}, x_{j}\right) \bar{n}_{j}(x) s_{j}\left(x_{j}^{\prime}, x\right) \prod_{i=1, i \neq j}^{N} \delta\left(x_{i}^{\prime}-x_{i}\right)
$$

(Dieckmann 1994; Dieckmann et al. 1995; Dieckmann and Law 1996). Here the product of Dirac delta functions captures the fact that all but the $j^{t h}$ component of $x$ remain unchanged, while the summation adds the transition rates for those $j^{\text {th }}$ components across all species. 
Based on these transition rates, the master equation for the probability $P(x)$ of observing trait value $x$,

$$
\frac{\mathrm{d}}{\mathrm{d} t} P(x)=\int\left[r\left(x, x^{\prime}\right) P\left(x^{\prime}\right)-r\left(x^{\prime}, x\right) P(x)\right] \mathrm{d} x^{\prime},
$$

then describes the directed evolutionary random walks in trait space resulting from sequences of trait substitutions.

Monomorphic Deterministic Model. If mutational steps $x_{i} \rightarrow x_{i}^{\prime}$ are small, the average of many realizations of the evolutionary random walk model described above is closely approximated by

$$
\frac{\mathrm{d}}{\mathrm{d} t} x_{i}=\int\left(x_{i}^{\prime}-x_{i}\right) r\left(x^{\prime}, x\right) \mathrm{d} x^{\prime}
$$

for $i=1, \ldots, N$ (e.g., van Kampen 1981). After inserting $r\left(x^{\prime}, x\right)$ as derived above, this yields

$$
\frac{\mathrm{d}}{\mathrm{d} t} x_{i}=\mu_{i}\left(x_{i}\right) \bar{b}_{i}\left(x_{i}, x\right) \bar{n}_{i}(x) \int s_{i}\left(x_{i}^{\prime}, x\right)\left(x_{i}^{\prime}-x_{i}\right) M_{i}\left(x_{i}^{\prime}, x_{i}\right) \mathrm{d} x_{i}^{\prime} .
$$

By expanding $s_{i}\left(x_{i}^{\prime}, x\right)=\max \left(0, \bar{f}_{i}\left(x_{i}^{\prime}, x\right)\right) / \bar{b}_{i}\left(x_{i}^{\prime}, x\right)$ around $x_{i}$ to first order in $x_{i}^{\prime}$, we obtain $s_{i}\left(x_{i}^{\prime}, x\right)=\max \left(0,\left(x_{i}^{\prime}-x_{i}\right) g_{i}(x)\right) / \bar{b}_{i}\left(x_{i}, x\right)$ with $g_{i}(x)=$ $\left.\frac{\partial}{\partial x_{i}^{\prime}} \bar{f}_{i}\left(x_{i}^{\prime}, x\right)\right|_{x_{i}=x_{i}^{\prime}}$; notice here that $\bar{f}_{i}\left(x_{i}, x\right)=0$. This means that in the $x_{i}^{\prime}$-integral above only half of the total $x_{i}^{\prime}$-range contributes, while for the other half the integrand is 0 . If mutation distributions $M_{i}$ are symmetric $M_{i}\left(x_{i}+\Delta x_{i}, x_{i}\right)=M_{i}\left(x_{i}-\Delta x_{i}, x_{i}\right)$ for all $i, x_{i}$, and $\Delta x_{i}$ - we obtain

$$
\frac{\mathrm{d}}{\mathrm{d} t} x_{i}=\frac{1}{2} \mu_{i}\left(x_{i}\right) \bar{n}_{i}(x) \int\left(x_{i}^{\prime}-x_{i}\right)^{T}\left(x_{i}^{\prime}-x_{i}\right) M_{i}\left(x_{i}^{\prime}, x_{i}\right) \mathrm{d} x^{\prime} g_{i}(x) .
$$

The integral is the variance-covariance matrix of the mutation distribution $M_{i}$ around trait value $x_{i}$, denoted by $\sigma_{i}^{2}\left(x_{i}\right)$. Hence we recover the canonical equation of adaptive dynamics (Dieckmann 1994; Dieckmann and Law 1996),

$$
\frac{\mathrm{d}}{\mathrm{d} t} x_{i}=\frac{1}{2} \mu_{i}\left(x_{i}\right) \bar{n}_{i}(x) \sigma_{i}^{2}\left(x_{i}\right) g_{i}(x)
$$

for $i=1, \ldots, N$. When mutational steps $x_{i} \rightarrow x_{i}^{\prime}$ are not small, higher-order correction terms can be derived: these improve the accuracy of the canonical equation and also cover non-symmetric mutation distributions (Dieckmann 1994; Dieckmann and Law 1996).

Polymorphic Deterministic Model. When mutation probabilities are high, evolution is no longer mutation-limited, so that the two classes of models introduced above - both being derived from the analysis of invasions into essentially monomorphic populations - cannot offer quantitatively accurate approximations of the underlying individual-based birth-death-mutation processes. Provided that population sizes are sufficiently large, it instead becomes 
appropriate to investigate the average distribution-valued dynamics of many realizations of the birth-death-mutation process,

$$
\frac{\mathrm{d}}{\mathrm{d} t} p(x)=\int\left[p^{\prime}(x)-p(x)\right] r\left(p^{\prime}, p\right) \mathrm{d} p^{\prime} .
$$

Inserting the transition rates $r\left(p^{\prime}, p\right)$ specified above for the individual-based evolutionary model, we can infer (by collapsing the integrals using the sifting properties of the Dirac delta function and of the generalized delta function)

$$
\frac{\mathrm{d}}{\mathrm{d} t} p_{i}(x)=r_{i}^{+}\left(x_{i}, p\right)-r_{i}^{-}\left(x_{i}, p\right)
$$

for $i=1, \ldots, N$. Inserting $r_{i}^{+}\left(x_{i}, p\right)$ and $r_{i}^{-}\left(x_{i}, p\right)$ from above, this gives

$$
\begin{aligned}
\frac{\mathrm{d}}{\mathrm{d} t} p_{i}(x)= & {\left[1-\mu_{i}\left(x_{i}\right)\right] b_{i}\left(x_{i}, p\right) p_{i}\left(x_{i}\right) } \\
& +\int \mu_{i}\left(x_{i}^{\prime}\right) b_{i}\left(x_{i}^{\prime}, p\right) p_{i}\left(x_{i}^{\prime}\right) M_{i}\left(x_{i}^{\prime}, x_{i}\right) \mathrm{d} x_{i}^{\prime}-d_{i}\left(x_{i}, p\right) p_{i}\left(x_{i}\right) .
\end{aligned}
$$

Further analysis is simplified by assuming that the mutation distributions $M_{i}$ are not only symmetric but also homogeneous $-M_{i}\left(x_{i}^{\prime}+\Delta x_{i}, x_{i}+\Delta x_{i}\right)=$ $M_{i}\left(x_{i}^{\prime}, x_{i}\right)$ for all $i, x_{i}^{\prime}, x_{i}$, and $\Delta x_{i}$. Expanding $\mu_{i}\left(x_{i}^{\prime}\right) b_{i}\left(x_{i}^{\prime}, p\right) p_{i}\left(x_{i}^{\prime}\right)$ up to second order in $x^{\prime}$ around $x_{i}$,

$$
\begin{aligned}
\mu_{i}\left(x_{i}^{\prime}\right) b_{i}\left(x_{i}^{\prime}, p\right) p_{i}\left(x_{i}^{\prime}\right)= & \mu_{i}\left(x_{i}\right) b_{i}\left(x_{i}, p\right) p_{i}\left(x_{i}\right)+\left(x_{i}^{\prime}-x_{i}\right) \frac{\partial}{\partial x_{i}} \mu_{i}\left(x_{i}\right) b_{i}\left(x_{i}, p\right) p_{i}\left(x_{i}\right) \\
& +\frac{1}{2}\left(x_{i}^{\prime}-x_{i}\right)^{T}\left[\frac{\partial^{2}}{\partial x_{i}^{2}} \mu_{i}\left(x_{i}\right) b_{i}\left(x_{i}, p\right) p_{i}\left(x_{i}\right)\right]\left(x_{i}^{\prime}-x_{i}\right),
\end{aligned}
$$

then yields

$$
\frac{\mathrm{d}}{\mathrm{d} t} p_{i}(x)=f_{i}\left(x_{i}, p\right) p_{i}\left(x_{i}\right)+\frac{1}{2} \sigma_{i}^{2}\left(x_{i}\right) * \frac{\partial^{2}}{\partial x_{i}^{2}} \mu_{i}\left(x_{i}\right) b_{i}\left(x_{i}, p\right) p_{i}\left(x_{i}\right),
$$

with $f_{i}\left(x_{i}, p\right)=b_{i}\left(x_{i}, p\right)-d_{i}\left(x_{i}, p\right), \sigma_{i}^{2}\left(x_{i}\right)=\int\left(x_{i}^{\prime}-x_{i}\right)^{T}\left(x_{i}^{\prime}-x_{i}\right) M_{i}\left(x_{i}^{\prime}, x_{i}\right)$ $\mathrm{d} x_{i}^{\prime}$, and with $*$ denoting the elementwise multiplication of two matrices followed by summation over all resultant matrix elements. This result also provides a good approximation when mutation distributions are heterogeneous, as long as $\sigma_{i}^{2}\left(x_{i}\right)$, rather than being strictly independent of $x_{i}$, varies very slowly with $x_{i}$ on the scale given by its elements.

\section{References}

1. Arditi, R., and Ginzburg, L.R. (1989). Coupling in predator-prey dynamics: Ratio-dependence. Journal of Theoretical Biology 139: 311-326

2. Athreya, K.B., and Ney, P.E. (1972). Branching Processes. New York, USA: Springer Verlag 
3. Brännström, Å., Loeuille, N., Loreau, M., and Dieckmann, U. Metabolic scaling, competition, and predation induce repeated adaptive radiation, in preparation

4. Bürger, R. (1998). Mathematical properties of mutation-selection models. Genetica 103: 279-298

5. Bush, G.L. (1969). Sympatric host race formation and speciation in frugivorous flies of the genus Rhagoletis (Diptera: Tephritidae). Evolution 23: 237-251

6. Caldarelli, G., Higgs, P.G., and McKane, A.J. (1998). Modelling coevolution in multispecies communities. Journal of Theoretical Biology 193: 345-358

7. Calow, P. (1999). Encyclopedia of Ecology and Environmental Management. Oxford, UK: Blackwell Publishing

8. Cheptou, P.O. (2004). Allee effect and self-fertilization in hermaphrodites: Reproductive assurance in demographically stable populations. Evolution 58: 2613-2621

9. Christiansen, F.B. (1991). On conditions for evolutionary stability for a continuously varying character. American Naturalist 138: 37-50

10. Clements, F.E. (1916). Plant succession: An analysis of the development of vegetation. Publication No. 242. Carnegie Institute Washington, Washington, D.C., USA

11. Crank, J. (1975). The Mathematics of Diffusion. Oxford, UK: Clarendon Press

12. Cressman, R. (1990). Evolutionarily stable strategies depending on population-density. Rocky Mountain Journal of Mathematics 20: 873-877

13. Crow, J.F., and Kimura, M. (1970). An Introduction to Population Genetics Theory. New York, USA: Harper and Row

14. Dercole, F., and Rinaldi, S. (2002). Evolution of cannibalistic traits: Scenarios derived from adaptive dynamics. Theoretical Population Biology 62: 365-374

15. Dercole, F., Ferrière, R., and Rinaldi, S. (2002). Ecological bistability and evolutionary reversals under asymmetrical competition. Evolution 56: 10811090

16. Dieckmann, U. (1994). Coevolutionary Dynamics of Stochastic Replicator Systems. Jülich, Germany: Central Library of the Research Center Jülich

17. Dieckmann, U., and Doebeli, M. (1999). On the origin of species by sympatric speciation. Nature 400: 354-357

18. Dieckmann, U., and Ferrière, R. (2004). Adaptive dynamics and evolving biodiversity. In: Ferrière, R., Dieckmann, U., and Couvet, D., eds. Evolutionary Conservation Biology. Cambridge, UK: Cambridge University Press, pp. 188224

19. Dieckmann, U., and Law, R. (1996). The dynamical theory of coevolution: A derivation from stochastic ecological processes. Journal of Mathematical Biology 34: 579-612

20. Dieckmann, U., Doebeli, M., Metz, J.A.J., and Tautz, D. eds. (2004). Adaptive Speciation. Cambridge, UK: Cambridge University Press

21. Dieckmann, U., Marrow, P., and Law, R. (1995). Evolutionary cycling in predator-prey interactions: Population dynamics and the Red Queen. Journal of Theoretical Biology 176: 91-102

22. Diehl, S., and Feissel, M. (2000). Effects of enrichment on three-level food chains with omnivory. American Naturalist 155: 200-218

23. Doebeli, M., and Dieckmann, U. (2000). Evolutionary branching and sympatric speciation caused by different types of ecological interactions. American Naturalist 156: S77-S101 
24. Doebeli, M., and Dieckmann, U. (2003). Speciation along environmental gradients. Nature 421: 259-264

25. Doebeli, M., and Dieckmann, U. (2005). Adaptive dynamics as a mathematical tool for studying the ecology of speciation processes. Journal of Evolutionary Biology 18: 1194-1200

26. Doebeli, M., Dieckmann, U., Metz, J.A.J., and Tautz, D. (2005). What we have also learned: Adaptive speciation is theoretically plausible. Evolution 59: 691-695

27. Drake, J.A. (1990). Communities as assembled structures: Do rules govern pattern? Trends in Ecology and Evolution 5: 159-164

28. Drossel, B., and McKane, A.J. (2000). Competitive speciation in quantitative genetic models. Journal of Theoretical Biology 204: 467-478

29. Drossel, B., Higgs, P.G., and McKane, A.J. (2001). The influence of predatorprey population dynamics on the long-term evolution of food web structure. Journal of Theoretical Biology 208: 91-107

30. Eliot, C. Method and metaphysics in Clements's and Gleason's ecological explanations. Studies in History and Philosophy of Science Part C: Studies in History and Philosophy of Biological and Biomedical Sciences, in press (available online at http://people.hofstra.edu/faculty/Christopher_H_Eliot/EliotClements.pdf)

31. Elton, C.S. (1958). Ecology of invasions by animals and plants. London, UK: Chapman and Hall

32. Ernande, B., Dieckmann, U., and Heino, M. (2002). Fisheries-induced changes in age and size at maturation and understanding the potential for selectioninduced stock collapse. ICES CM 2002/Y:06

33. Eshel, I. (1983). Evolutionary and continuous stability. Journal of Theoretical Biology 103: 99-111

34. Felsenstein, J. (1981). Skepticism towards Santa Rosalia, or Why are there so few kinds of animals? Evolution 35: 124-238

35. Ferrière, R. (2000). Adaptive responses to environmental threats: Evolutionary suicide, insurance, and rescue. International Institute for Applied Systems Analysis, Laxenburg, Austria: Options Spring 2000, pp. 12-16

36. Ferrière, R., Bronstein, J.L., Rinaldi, S., Gauduchon, M., and Law, R. (2002). Cheating and the evolutionary stability of mutualism. Proceedings of Royal Society of London Series B 269: 773-780

37. Fisher, R.A. (1930). The Genetical Theory of Natural Selection. Oxford, UK: Clarendon Press

38. Geritz, S.A.H., and Kisdi, É. (2000). Adaptive dynamics in diploid sexual populations and the evolution of reproductive isolation. Proceedings of the Royal Society of London B 267: 1671-1678

39. Geritz, S.A.H., Gyllenberg, M., Jacobs, F.J.A., and Parvinen, K. (2002) Invasion dynamics and attractor inheritance. Journal of Mathematical Biology 44: $548-560$

40. Geritz, S.A.H., Kisdi, É., Meszéna, G., and Metz, J.A.J. (1998). Evolutionary singular strategies and the adaptive growth and branching of the evolutionary tree. Evolutionary Ecology 12: 35-57

41. Geritz, S.A.H., Kisdi, É., Meszéna, G., and Metz, J.A.J. (2004). Adaptive dynamics of speciation: Ecological underpinnings. In: Dieckmann, U., Doebeli, M., Metz, J.A.J., and Tautz, D. eds. Adaptive Speciation. Cambridge, UK: Cambridge University Press, pp. 54-75 
42. Geritz, S.A.H., Metz, J.A.J., Kisdi, É., and Meszéna, G. (1997). Dynamics of adaptation and evolutionary branching. Physical Review Letters 78: 2024-2027

43. Gillespie, D.T. (1976). A general method for numerically simulating the stochastic time evolution of coupled chemical reactions. Journal of Computational Physics 22: 403-434

44. Gleason, H.A. (1926). The individualistic concept of the plant association. Bulletin of the Torrey Botanical Club 53: 7-26

45. Gyllenberg, M., and Parvinen, K. (2001). Necessary and sufficient conditions for evolutionary suicide. Bulletin of Mathematical Biology 63: 981-993

46. Gyllenberg, M., Parvinen, K., and Dieckmann, U. (2002). Evolutionary suicide and evolution of dispersal in structured metapopulations. Journal of Mathematical Biology 45: 79-105

47. Haldane, J.B.S. (1932). The Causes of Evolution. London, UK: Harper

48. Hardin, G. (1968). The tragedy of the commons. Science 162: 1243-1248

49. HilleRisLambers, R., and Dieckmann, U. (2003). Competition and predation in simple food webs: Intermediately strong trade-offs maximize coexistence. Proceedings of the Royal Society of London B 270: 2591-2598

50. HilleRisLambers, R., and Dieckmann, U. Evolving Omnivory: Restrictions on simple food webs imposed by the interplay between ecology and evolution, submitted

51. Holt, R.D., and Polis, G.A. (1997). A theoretical framework for intraguild predation. American Naturalist 149: 745-764

52. Hubbell, S.P. (2001). The Unified Neutral Theory of Biodiversity and Biogeography. Princeton, USA: Princeton University Press

53. Ito, H., and Ikegami, T. (2003). Evolutionary dynamics of a food web with recursive branching and extinction. In: Standish, R.K., Bedau, M.A., and Abbass, H.A. eds. Artificial Life VIII, Cambridge, USA: MIT Press, pp. 207-215

54. Ito, H.C., and Ikegami, T. (2006). Food web formation with recursive evolutionary branching. Journal of Theoretical Biology 238: 1-10

55. Johnson, P.A., Hoppensteadt, F.C., Smith, J.J., and Bush, G.L. (1996). Conditions for sympatric speciation: A diploid model incorporating habitat fidelity and non-habitat assortative mating. Evolutionary Ecology 10: 187-205

56. Kimura, M. (1965). A stochastic model concerning maintenance of genetic variability in quantitative characters. Proceedings of the National Academy of Sciences of the USA 54: 731-735

57. Kirkpatrick, M. (1996). Genes and adaptation: A pocket guide to theory. In: Rose, M.R., and Lauder, G.V. eds. Adaptation. San Diego, USA: Academic Press, pp. 125-128

58. Kisdi, É., and Meszéna, G. (1993). Density-dependent life-history evolution in fluctuating environments. In: Yoshimura, J., and Clark, C. eds. Adaptation in a Stochastic Environment, Lecture Notes in Biomathematics 98. Berlin, Germany: Springer, pp. 26-62

59. Kokko, H., and Brooks, R. (2003). Sexy to die for? Sexual selection and the risk of extinction. Annales Zoologici Fennici 40: 207-219

60. Law, R. (1999). Theoretical aspects of community assembly. In: McGlade, J. ed. Advanced ecological theory: Principles and applications. Oxford, UK: Blackwell Science, pp. 143-171

61. Law, R., Marrow, P., and Dieckmann, U. (1997). On evolution under asymmetric competition. Evolutionary Ecology 11: 485-501 
62. Le Galliard, J.F., Ferrière, R., and Dieckmann, U. (2003). The adaptive dynamics of altruism in spatially heterogeneous populations. Evolution 57: 1-17

63. Levins, R. (1962). Theory of fitness in a heterogeneous environment. I. The fitness set and adaptive function. American Naturalist 96: 361-373

64. Levins, R. (1968). Evolution in Changing Environments. Princeton, USA: Princeton University Press

65. Loeuille, N., and Loreau, M. (2005). Evolutionary emergence of size-structured food webs. Proceedings of the National Academy of Sciences of the USA 102: 5761-5766

66. MacArthur, R., and Levins, R. (1967). The limiting similarity, convergence, and divergence of coexisting species. American Naturalist 101: 377-385

67. Matsuda, H. (1985). Evolutionarily stable strategies for predator switching. Journal of Theoretical Biology 115: 351-366

68. Matsuda, H., and Abrams, P.A. (1994a). Runaway evolution to self-extinction under asymmetrical competition. Evolution 48: 1764-1772

69. Matsuda, H., and Abrams, P.A. (1994b). Timid consumers - Self-extinction due to adaptive change in foraging and anti-predator effort. Theoretical Population Biology 45: 76-91

70. May, R.M. (1973). Stability and Complexity in Model Ecosystems. Princeton, USA: Princeton University Press

71. Maynard Smith, J. (1966). Sympatric speciation. American Naturalist 100: $637-650$

72. Maynard Smith, J. (1982). Evolution and the Theory of Games. Cambridge, UK: Cambridge University Press

73. Maynard Smith, J., and Price, G.R. (1973). Logic of animal conflict. Nature 246: $15-18$

74. Mayr, E. (1963). Animal Species and Evolution. Cambridge, USA: Harvard University Press

75. Mayr, E. (1982). The Growth of Biological Thought: Diversity, Evolution, and Inheritance. Cambridge, USA: The Belknap Press of Harvard University Press

76. McCann, K.S. (2000). The diversity-stability debate. Nature 405: 228-233

77. Meszéna, G., Kisdi, É., Dieckmann, U., Geritz, S.A.H., and Metz, J.A.J. (2001). Evolutionary optimisation models and matrix games in the unified perspective of adaptive dynamics. Selection 2: 193-210

78. Metz, J.A.J., Geritz, S.A.H., Meszéna, G., Jacobs, F.J.A., and van Heerwaarden, J.S. (1996). Adaptive dynamics: A geometrical study of the consequences of nearly faithful reproduction. In: van Strien, S.J., Verduyn Lunel, S.M. eds. Stochastic and Spatial Structures of Dynamical Systems. Amsterdam, The Netherlands: North-Holland, pp. 183-231

79. Metz, J.A.J., Nisbet, R.M., and Geritz, S.A.H. (1992). How should we define fitness for general ecological scenarios? Trends in Ecology and Evolution 7: 198-202

80. Meyer, A. (1993). Phylogenetic relationships and evolutionary processes in east African cichlid fishes. Trends in Ecology and Evolution 8: 279-284

81. Mylius, S.D., Klumpers, K., de Roos, A.M., and Persson, L. (2001). Impact of intraguild predation and stage structure on simple communities along a productivity gradient. American Naturalist 158: 259-276

82. Odling-Smee, F.J., Laland, K.N., and Feldman, M.W. (2003). Niche Construction: The Neglected Process in Evolution. Princeton, USA: Princeton University Press 
83. Oksanen, L., and Oksanen, T. (2000). The logic and realism of the hypothesis of exploitation ecosystems. American Naturalist 155: 703-723

84. Oksanen, L., Fretwell, S., Arruda, J., and Niemela, P. (1981). Exploitation ecosystems in gradients of primary productivity. American Naturalist 118: 240-261

85. Parvinen, K. (2006). Evolutionary suicide. Acta Biotheoretica 53: 241-264

86. Peters, R.H. (1983). The Ecological Implications of Body Size. Cambridge, UK: Cambridge University Press

87. Post, W.M., and Pimm, S.L. (1983). Community assembly and food web stability. Mathematical Biosciences 64: 169-192

88. Press, W.H., Teukolsky, S.A., Vetterling, W.T., and Flannerty, B.P. (1992). Numerical Recipes in C: The Art of Scientific Computing, 2nd edition. Cambridge, USA: Cambridge University Press

89. Rankin, D.J., and López-Sepulcre, A. (2005). Can adaptation lead to extinction? Oikos 111: 616-619

90. Rosenzweig, M.L. (1978). Competitive speciation. Biological Journal of the Linnean Society 10: 275-289

91. Roughgarden, J. (1974) Species packing and the competition function with illustrations from coral reef fish. Theoretical Population Biology 5: 163-186

92. Roughgarden, J. (1979). Theory of Population Genetics and Evolutionary Ecology: An Intro-duction. New York, USA: Macmillan

93. Roughgarden, J. (1983). The theory of coevolution. In: Futuyma, D.J., and Slatkin, M. eds. Coevolution. Sunderland, USA: Sinauer Associates, pp. 33-64

94. Rummel, J.D., and Roughgarden, J. (1985). A theory of faunal build-up for competition communities. Evolution 39:1009-1033

95. Schliewen, U.K., Tautz, D., and Pääbo, S. (1994). Sympatric speciation suggested by monophyly of crater lake cichlids. Nature 368: 629-632

96. Schluter, D. (2000). The Ecology of Adaptive Radiation. Oxford, UK: Oxford University Press

97. Taper, M.L., and Case, T.J. (1992). Coevolution among competitors. In: Futuyma, D., and Antonivics, J. eds. Oxford Surveys in Evolutionary Biology, Volume 8. Oxford, UK: Oxford University Press, pp. 63-111

98. Tilman, D. (1982). Resource Competition and Community Structure. Princeton, USA: Princeton University Press

99. Udovic, D. (1980). Frequency-dependent selection, disruptive selection, and the evolution of reproductive isolation. American Naturalist 116: 621-641

100. van Kampen, N.G. (1992). Stochastic Processes in Physics and Chemistry. Amsterdam, The Netherlands: North-Holland

101. van Tienderen, P.H., and de Jong, G. (1986). Sex-ratio under the haystack model - Polymorphism may occur. Journal of Theoretical Biology 122: 69-81

102. Vandermeer, J.H. (1970). The community matrix and the number of species in a community. American Naturalist 104: 73-83

103. Webb, C.T. (2003). A complete classification of Darwinian extinction in ecological interactions. American Naturalist 161: 181-205

104. Wright, S. (1932). The roles of mutation, inbreeding, crossbreeding and selection in evolution. Proceedings of the 6th International Congress of Genetics 1: 356-366

105. Wright, S. (1967). Surfaces of selective value. Proceedings of the National Academy of Sciences of the USA 102: 81-84 